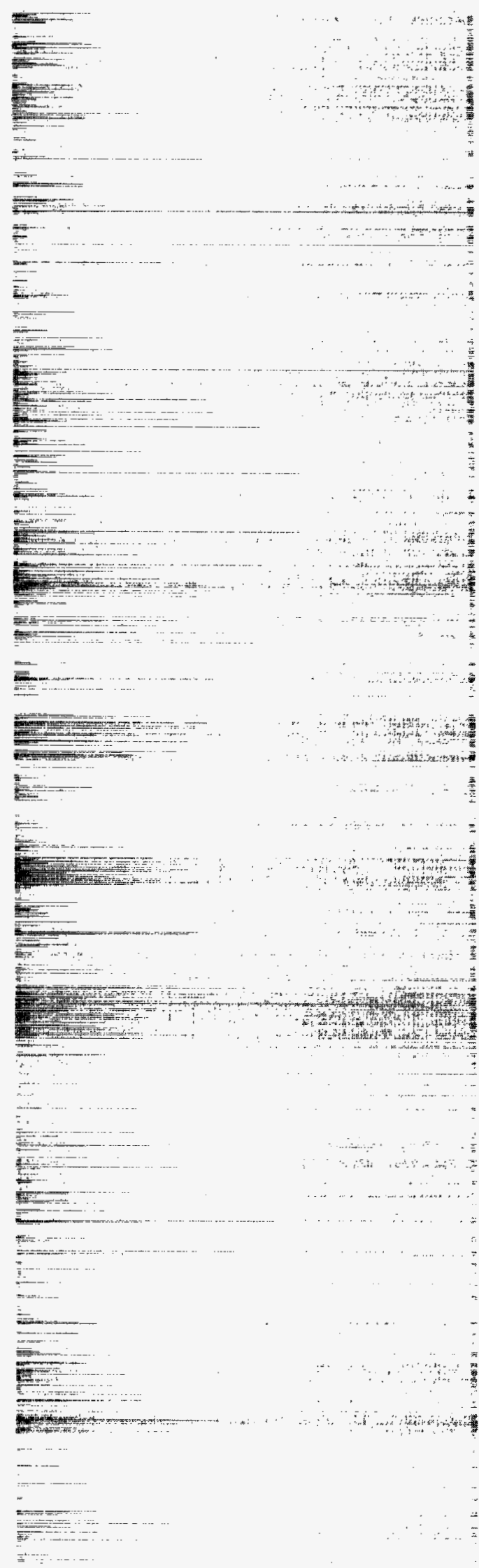

\title{
Using Conversions of Chemically Reacting \\ Tracers for Numerical Determination of \\ Temperature Profiles in Flowing Systems and \\ Temperature Histories in Batch Systems
}

\section{DISCLAIMER}

This report was prepared as an account of work sponsored by an agency of the United States Government. Neither the United States Government nor any agency thereof, nor any of their employees, makes any warranty, express or implied, or assumes any legal liability or responsibility for the accuracy, completeness, or usefulness of any information, apparatus, product, or process disclosed, or represents that its use would not infringe privately owned rights. Reference herein to any specific commercial product, process, or service by trade name, trademark, manufacturer, or otherwise does not necessarily constitute or imply its endorsement, recommendation, or favoring by the United States Government or any agency thereof. The views and opinions of authors expressed herein do not necessarily state or reflect those of the United States Government or any agency thereof.

\section{Los Alamos \\ NATIONAL LABOAATORY}

Los Alamos National Laboratory is operated by the University of California for the United States Department of Energy under contract W-7405-ENG-36. 
An Affirmative Action/Equal Opportunity Employer

This report was prepared as an account of work sponsored by an agency of the United States Government. Neither The Regents of the University of California, the United States

Government nor any agency thereof, nor any of their emiployees, makes any warranty, express or implied, or assumes any legal liability or responsibility for the accuracy, completeness, or usefulness of any information, apparatus, product, or process disclosed, or represents that its use would not infringe privately owned rights. Reference herein to any specific commercial product, process, or service by trade name, trademark, manufacturer, or otherwise, does not necessarily constitute or imply its endorsement, recommendation, or favoring by The Regents of the University of California, the United States Government, or any agency thereof. The views and opinions of authors expressed herein do not necessarily state or reflect those of The Regents of the University of California, the United States Government, or any agency thereof. The Los Alamos National Laboratory strongly supports acndemic freedom and a researcher's right to publish; therefore, the Laboratory as an institution does not endorse the viewpoint of a publication or guarantee its technical correctness. 
Using Conversions of Chemically Reacting

Tracers for Numerical Determination of

Temperature Profiles in Flowing Systems and

Temperature Histories in Batch Systems

Lee F. Brown

Rajeev M. Chemburkar

Bruce A. Robinson

Bryan J. Travis 


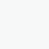




\section{CONTENTS}

LIST OF TABLES vii

LIST OF FIGURES vii

PREFACE $\quad$ ix

NOMENCLATURE $\quad x$

ACRONYMS

ABSTRACT 1

I. EXECUTIVE SUMMARY 1

II. INTRODUCTION $\quad 3$

A. Need for Indirect Measurements of Temperatures within Flowing

B. Need for Temperature Histories of Batch Systems 4

C. Need for Indirect Temperature Measurements in Hot Dry Rock
Geothermal Reservoirs

D. The Possibility of Measuring Temperatures Using Chemical
Reactions

E. Plug-Flow, Dispersed-Flow, Perfectly Mixed Batch, and
Imperfectly Mixed Batch Systems

III. THE FUNDAMENTAL RELATIONSHIPS $\quad 6$

A. Basic Assumptions for Batch Systems 6

B. Derivation of the Fundamental Relationship for Batch Systems 7

C. Basic Assumptions for Flowing Systems 7

D. Derivation of the Fundamental Relationship for Flowing $\begin{array}{ll}\text { Systems. } & 8 \\ \text { Implications of the Fundamental Relationships } & 9\end{array}$

E. Implications of the Fundamental Relationships
1. Identical nature of the two fundamental relationships

2. The experimental variable for multi-reaction tracer tests 9

3. Suitable classes of reactions for reacting tracer tests 9

4. Pulse and continuous injection of reacting tracers into
flowing systems

F. Use of First-Order Reactions 10

IV. SINGLE-REACTION TRACER TESTS 11 
V. EQUATIONS FOR MULTIPLE-REACTION TRACER TESTS OF

$\begin{array}{ll}\text { MONOTONIC SYSTEMS } & 12 \\ \text { A. Forms of Equations Considered } & 12\end{array}$

$\begin{array}{ll}\text { 1. Equations for batch and plug-flow systems } & 12\end{array}$

2. Fredholm integral equations of the first kind 13

B. A Fredholm Equation Using a Simple Temperature-Distribution
Function

C. A Fredholm Equation with a Cumulative Temperature-Distribution
Function

D. A Fredholm Equation Containing a Series Expansion I_eading to
an Iterative Solution

E. An Equation Used for Parameter Optimization $\quad 15$

1. Function to be minimized 15

$\begin{array}{ll}2 . & \text { The optimization procedure } \\ \end{array}$

VI. EQUATIONS FOR SYSTEMS CONTAINING TEMPERATURE EXTREMA 16

A. The Equations for Monotonic Systems 16

B. Distribution Functions in Systems Containing Temperature Extrema 17

C. Multiple Solutions in Systems Containing Temperature Extrema 18

VII. SOLUTION PROCEDURES FOR THE FREDHOLM EQUATIONS · $\quad 18$

A. Difficulties Inherent in Inverting First-Kind Fredholm Equations 18

$\begin{array}{ll}\text { B. A General Method of Regularization That Can Incorporate } & 19 \\ \text { Constraints and Boundary Conditions } & \end{array}$

C. The Butler, Reeds, and Dawson Method Incorporating the
Non-Negativity Constraint

D. An Iterative Method That Gives The Smoothest Solution at Any
Desired Accuracy

VIII. TESTING THE EQUATIONS AND SOLUTION PROCEDURES

A. Three Typical Temperature-Profile Functions 21

$\begin{array}{ll}\text { 1. Analytic expressions for three temperature-profile types } & 21\end{array}$

2. The simple temperature distributions for the three typical
temperature profiles

3. The cumulative temperature distributions for the three
typical temperature profiles

B. Inverting the Fredholm Equations 23

1. The three Fredholm equations for obtaining temperature
profiles

2. Approach to testing the different factors 24

3. Effect of activation energies 24

4. Effect of number of reacting tracers 25

5. Effect of error level in the experimental data 25

6. Comparing the use of the three equations 26

7. Temperature profiles exhibiting a maximum 31

$\begin{array}{ll}\text { IX. DISCUSSION } & 34\end{array}$ 
X. PRACTICAL IMPLEMENTATION OF THE PRINCIPLES

A. Characteristics of Suitable Tracers 35

B. Temperatures and Temperature Measurement in Hot Dry Rock Geothermal Reservoirs

$\begin{array}{lll}\text { XI. CONCLUSIONS } & 36\end{array}$

$\begin{array}{ll}\text { XII. ACKNOWLEDGMENTS } & 38\end{array}$

$\begin{array}{ll}\text { XIII. REFERENCES } & 38\end{array}$

\section{APPENDIXES}

A. Parameter Precision in Least-Squares Optimization

B. Development of the Fredholm Equation Containing the Simple

Temperature Distribution Function for Systems with

Temperature Extrema

C. Expressions for the Matrices and Vector in Eq. (VII-5)

\section{LIST OF TABLES}

Table 1. Characteristics of profiles with extrema used for testing

Table 2. Comparison of data and RHS of Eq. (III-14) in attempt to recover Profile C3 pictured in Fig. 13.

\section{LIST OF FIGURES}

Figure 1. Step-function approximation to an S-shaped temperature profile.

Figure 2. Types of temperature profiles for typical flowing systems. A. Adiabatic reactor with exothermic reaction or Hot Dry Rock reservoir with conduction-limited heat transfer. B. Highly fractured Hot Dry Rock reservoir. C. Plug-flow chemical reactor with exothermic reaction and external heat exchange.

Figure 3. Simple temperature distributions corresponding to profiles in Fig. 2. A. Adiabatic reactor with exothermic reaction or Hot Dry Rock reservoir with conductionlimited heat transfer. B. Highly fractured Hot Dry Rock reservoir. C. Plug-flow chemical reactor with exothermic reaction and external heat exchange.

Figure 4. Cumulative temperature distributions corresponding to profiles in Fig. 2.

A. Adiabatic reactor with exothermic reaction or Hot Dry Rock reservoir with conduction-limited heat transfer. B. Highly fractured Hot Dry Rock reservoir.

C. Plug-flow chemical reactor with exothermic reaction and external heat exchange.

Figure 5: Effect of range of activation energy when temperature follows Profile $B$.

Error $=1 \%$, tolerance $=0.01, q_{0}=q_{1}=1$, and $\alpha=1$. Solid curve shows the actual temperature profile and dashed curve depicts the initial estimate. Squares, triangles, X's, +'s, and circles represent the results obtained for activation-energy ranges of $20-80,80-140,140-200,40-200$, and $40-46 \mathrm{~kJ} / \mathrm{mol}$, respectively. 
Figure 6: Effect of number of data points on the results. Error $=1 \%$, tolerance $=0.01$, $\mathrm{q}_{0}=\mathrm{q}_{1}=1$, and $\alpha=1$. Solid curve shows the actual temperature profile and dashed curve depicts the initial estimate. Squares, triangles, $\mathrm{XXX},++$, and circles represent the results obtained for $n=2,3,5,7$, and 13 , respectively.

Figure 7: (a) Effect of error when temperature follows Profile A. (b) Discrimination between two rival profiles under various levels of error. $E_{\min }=40 \mathrm{~kJ} / \mathrm{mol}$, $E_{\max }=80 \mathrm{~kJ} / \mathrm{mol}, \mathrm{n}=5, q_{0}=q_{1}=1$, and $\alpha=1$.

Figure 8: Effect of tolerance level on the results. $E_{\min }=20 \mathrm{~kJ} / \mathrm{mol}, E_{\max }=80 \mathrm{~kJ} / \mathrm{mol}$, $\mathrm{n}=7$, error $=1 \%, \mathrm{q}_{0}=\mathrm{q}_{\mathrm{l}}=1$, and $\alpha=1$. Solid curve shows the actual temperature profile and dashed curve depicts the initial estimate. Squares, triangles, and circles represent the results obtained for tolerances $=1 \%, 0.5 \%$, and $0.1 \%$ respectively. The number of iterations required in the three cases were 4,4 , and 10 , respectively.

Figure 9: Effect of non-negativity constraint on the results from Method 2 for Profile A. Solid curve denotes the true profile whereas dotted, dashed, and chain dot curves show the results using the Butler algorithm, general regularization with boundary conditions, and general regularization without boundary conditions, respectively. $\left(q_{0}=q_{l}=1, n=7, E_{\min }=20 \mathrm{~kJ} / \mathrm{mol}, E_{\max }=80 \mathrm{~kJ} / \mathrm{mol}\right.$, error $=0.1 \%$.

Figure 10: Comparison between the first-order regularization (empty symbols) and the Butler algorithm (solid symbols). ( $\mathrm{q}_{0}=\mathrm{q}_{1}=1, \mathrm{n}=7, \mathrm{E}_{\min }=20 \mathrm{~kJ} / \mathrm{mol}$, $\mathrm{E}_{\max }=80 \mathrm{~kJ} / \mathrm{mol}$, error $=0.1 \%$.)

Figure 11: Comparison of various methods. Solid curves depict the actual temperature profile. Dashed and dotted curves show the results obtained by Methods 1 and 2, respectively. Triangles represent the results of traditional regularization approach (Method 3), whereas circles represent the results of Butler approach (Method 3) without the non-negativity criterion. $E_{\min }=40 \mathrm{~kJ} / \mathrm{mol}, E_{\max }=80 \mathrm{~kJ} / \mathrm{mol}$, error $=3 \%$; for Method 3, tolerance $=0.01, q_{0}=q_{1}=1$, and $\alpha=1$.

Figure 12: Effect of the regularization parameter $\alpha$ on the results from Method 3. $\left(q_{0}=q_{1}=1, n=7, E_{\min }=20 \mathrm{~kJ} / \mathrm{mol}, E_{\max }=80 \mathrm{~kJ} / \mathrm{mol}\right.$, error $=0.1 \%$.)

Figure 13: Inversion of temperature profiles exhibiting peaks. Empty squares, circles, and solid squares represent results for Profiles $\mathrm{C1}, \mathrm{C} 2$, and $\mathrm{C} 3$, respectively.

$E_{\min }=40 \mathrm{~kJ} / \mathrm{mol}, E_{\max }=80 \mathrm{~kJ} / \mathrm{mol}$, error $=1 \%$, and $q_{0}=q_{1}=1$, and $\alpha=1$.

Figure 14: Results for Temperature Profile C3, obtained using various initial estimates.

Figure 15: Performance of Method 3 with poor initial estimates in the cases of Temperature Profiles A and B. Squares and triangles represent results obtained from initial profiles IC-A and IC-B, respectively. $n=7$, tolerance $=0.005, E_{\min }=20 \mathrm{~kJ} / \mathrm{mol}$, $E_{\max }=80 \mathrm{~kJ} / \mathrm{mol}, \mathrm{q}_{0}=1, \mathrm{q}_{1}=0, \alpha=100$. 


\section{PREFACE}

A significant portion of the material in this report has appeared in a publication (Chemburkar et al. 1991) that was condensed from an early draft of this report. The paper treated theoretical aspects of indirect temperature measurement in flowing systems using chemically reacting tracers. Material in the present report involving temperature histories of batch systems, thermal aspects of Hot Dry Rock geothermal systems, and experiments associated with Hot Dry Rock systems did not appear in the published paper. Using single-reaction tracer tests to give information about internal temperature patterns is also investigated here but not in the paper. Another subject not treated in the paper but discussed in this report is using parameter optimization to give approximate solutions to the equations for determining temperature patterns and histories from tracer tests. 


\section{NOMENCLATURE}

\section{$\underline{\text { Roman }}$}

A Pre-exponential factor in reaction-rate constant, $\left(\mathrm{m}^{3} / \mathrm{mol}\right)^{\mathrm{n}-1} \mathrm{~s}^{-1}$.

$\mathrm{A}_{\mathrm{f}} \quad$ Surface area of fracture, $\mathrm{m}^{2}$.

a Lower limit of intrinsic system variable.

$a_{r} \quad$ Fracture width in rock, $m$.

b Upper limit of intrinsic system variable.

C Concentration, $\mathrm{mol} / \mathrm{m}^{3}$.

c Lower limit of experimental-variable range.

$c_{p} \quad$ Heat capacity of flowing fluid, $\mathrm{J} /(\mathrm{g})(\mathrm{K})$.

d Upper limit of experimental-variable range.

E Activation energy of chemical reaction, $\mathrm{J} / \mathrm{mol}$.

f Indicates function of variable in parentheses immediately following. In Fredholm integral equations, it denotes a distribution function of the intrinsic system variable.

$f(C)$ Reaction-rate expression without principal rate constant, e.g., for $n^{\text {th }}$ order reaction, $f(C)=C^{n}$; for Langmuir-Hinshelwood bimolecular surface reaction, $f(C)=C_{A} C_{B} /\left(1+K_{A} C_{A}+K_{B} C_{B}\right)$.

$\mathrm{g}$ Experimental observations; function of experimental variable $\mathrm{x}$ or $\mathrm{E}$; often equal to $-\left(\mathrm{q} / \mathrm{A}_{\mathrm{i}} \mathrm{V}_{\mathrm{s}}\right) \ln \left(\mathrm{C}_{\mathrm{i} /} / \mathrm{C}_{\mathrm{io}}\right)$ or $-\left(1 / \mathrm{A}_{\mathrm{i}} \mathrm{t}_{\mathrm{p}}\right) \ln \left(\mathrm{C}_{\mathrm{if}} / \mathrm{C}_{\mathrm{io}}\right)$.

$\mathrm{K}$ Adsorption constant in Langmuir-Hinshelwood rate expression, $\mathrm{m}^{3} / \mathrm{mol}$.

$\mathrm{K}$ Kernel of integral, function of both experimental and intrinsic system variables.

k Reaction-rate constant, $\left(\mathrm{m}^{3} / \mathrm{mol}\right)^{\mathrm{n}-1} \mathrm{~s}^{-1}$.

m Number of mesh points specified when inverting Fredholm integral equations, dimensionless; also number of undetermined parameters in postulated function form of temperature profile, dimensionless.

n number of tracer reactions carried out in experiment, dimensionless; also order of reaction, dimensionless.

q Volumetric flow rate, $\mathrm{m}^{3} / \mathrm{s}$.

R Universal gas constant, $\mathrm{J} /(\mathrm{mol})(\mathrm{K})$.

rms Root-mean-square; refers to error levels imposed on data.

r Reaction rate, $\mathrm{mol} /\left(\mathrm{m}^{3}\right)(\mathrm{s})$.

s Dummy variable, $\mathrm{K}$.

T Temperature, $\mathrm{K}$.

t Time, $\mathbf{s}$.

$t_{\mathrm{g}} \quad$ Time since beginning of geothermal exploitation, $s$.

$\mathrm{U} \quad$ A temperature whose profile gives the same experimental observations as the true profile, $\mathrm{K}$.

$\mathrm{V}$ Volume, $\mathrm{m}^{3}$.

$\mathrm{x} \quad$ Experimental variable, various dimensions. 
y Intrinsic system variable, various dimensions.

Greek

$\alpha \quad$ Adjustable smoothing parameter in regularization method for inverting first-kind Fredholm integral equations, dimensionless.

$\beta \quad$ Undetermined parameter in parameter optimization equation, various dimensions.

H Cumulative temperature distribution function, dimensionless.

$\mathrm{H}(\mathrm{T}) \equiv \int_{0}^{\mathrm{T}} \eta(\mathrm{T}) \mathrm{dT} \quad$ or $\quad \int_{\mathrm{T}_{\min }}^{\mathrm{T}} \eta(\mathrm{T}) \mathrm{dT}$

$\eta \quad$ Temperature distribution within flowing system, $\mathrm{K}^{-1}$. The product $\eta(\mathrm{T}) \delta \mathrm{T}$ is the fraction of the system volume with temperatures between $T$ and $T+\delta T$.

$\lambda \quad$ Thermal conductivity, $\mathrm{J} /(\mathrm{s})(\mathrm{m})(\mathrm{K})$.

$\xi \quad$ Dimensionless time, $t / t_{p}$, dimensionless volume, $V / N_{s}$, or dimensionless length, $z / L$.

$\rho \quad$ Density of fluid, $\mathrm{g} / \mathrm{m}^{3}$.

$\tau \quad$ residence time or space time in flowing system, $\mathrm{s}$.

$\phi \quad$ Function to be minimized in optimization of undetermined parameters, usually dimensionless.

\section{Subscripts}

A Of substance A or of section A in reactor.

B Of substance $B$ or of section $B$ in reactor.

f Final value or value at sampling point

i Of reaction $i$ or of substance $i$.

$j$ Of reaction $j$, of substance $j$, or referring to the $j^{\text {th }}$ parameter.

max Maximum value in system.

min Minimum value in system.

p Of the period being considered.

r Of the rock.

s Of the system, or the amount of the subscripted variable traversed by the segment of fluid while traveling from the injection point to the withdrawal point.

0 Initial value, initial function, or value at point of injection.

\section{ACRONYMS}

BRD Butler-Reeds-Dawson; refers to developments in the paper by Butler et al. (1981).

FIEFK Fredholm integral equation of the first kind.

HDR Hot Dry Rock; a process for extracting geothermal energy.

LHS Left-hand side of an equation.

RHS Right-hand side of an equation. 


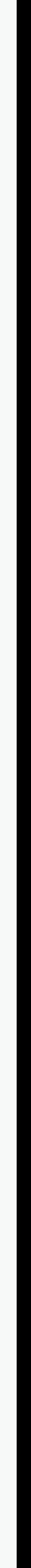




\title{
USING CONVERSIONS OF CHEMICALLY REACTING TRACERS FOR NUMERICAL DETERMINATION OF TEMPERATURE PROFILES IN FLOWING SYSTEMS AND TEMPERATURE HISTORIES IN BATCH SYSTEMS
}

\author{
by \\ Lee F. Brown, Rajeev M. Chemburkar, Bruce A. Robinson, and Bryan J. Travis
}

\begin{abstract}
This report presents the mathematical bases for measuring internal temperatures within batch and flowing systems using chemically reacting tracers. This approach can obtain temperature profiles of plugflow systems and temperature histories within batch systems. The differential equations for reactant conversion can be converted into Fredholm integral equations of the first kind. The experimental variable is the tracer-reaction activation energy. When more than one tracer is used, the reactions must have different activation energies to gain information. In systems with temperature extrema, multiple solutions for the temperature profiles or histories can exist.
\end{abstract}

When a single parameter in the temperature distribution is needed, a single-tracer test may furnish this information.

For multi-reaction tracer tests, three Fredholm equations are developed. Each of these has a different temperature distribution function that characterizes the internal temperature history or profile of the system. In the equations, the temperature distribution functions are the unknowns and must be determined. Two of the equations lend themselves to a widely used form of the regularization method of solution. The third distribution function is embedded within a new iterative approach to solving Fredholm equations by regularization. The iterative approach successfully overcomes the usual difficulty of determining the optimal value of the regularization smoothing parameter. Numerical tests show advantages and disadvantages of the three approaches. Effects of tracer-reaction activation energy, number of tracers used, and error in the data are evaluated. The methods can determine temperature histories and profiles for many existing systems, and can be a basis for analysis of the more complicated dispersed-flow systems.

An alternative to using the Fredholm-equation approach is the use of an assumed temperaturedistribution function and incorporation of this function into the basic integral equation describing tracer behavior. The function contains adjustable parameters which are optimized to give the temperature distribution. The assumed function requires a prejudgment of the form of the temperature distribution, which under some circumstances is a major drawback of this approach.

The iterative Fredholm equation method is tested to see what is required to discriminate between two models of the temperature behavior of Hot Dry Rock (HDR) geothermal reservoirs. Using as few as two reacting tracers can distinguish between the models and provide a reasonable approximation of the temperature profile within a reservoir. Experimentally, ester and amide hydrolyses are valid HDR tracer reactions for measuring temperatures in the range $75-100^{\circ} \mathrm{C}$. Hydrolyses of bromobenzene derivatives are valid $\mathrm{HDR}$ tracer reactions for measuring temperatures in the range $150-275^{\circ} \mathrm{C}$.

\section{EXECUTIVE SUMMARY}

Internal temperature characteristics of flowing systems and temperature histories of batch systems may be important to their analysis. Yet in many processes involving such systems, it is infeasible to use direct temperature probes to characterize the temperature field or history. Thus, the development of an indirect technique would be an important contribution. An important example of such a system is a Hot 
Dry Rock (HDR) geothermal reservoir, which extracts heat from the rock by passing a cold fluid from an injection well through the hot reservoir and out a withdrawal well. The system needs temperature measurements within the reservoir for models predicting reservoir life.

This report presents the mathematical bases of a method for obtaining temperature profiles in flowing systems and temperature histories of batch systems. It is proposed to measure internal temperatures using chemically reacting tracers. This report considers plug-flow (or piston-flow) and perfectly mixed batch systems. Systems of this type are met frequently, so the techniques developed here can be used directly. In addition, the development of the techniques for these systems provides a basis for treatment of the more complicated dispersed-flow and imperfectly mixed batch systems.

The equation describing the conversion of a chemically reacting tracer in a plug-flow system is mathematically identical with that describing the conversion in a perfectly mixed batch system. The two equations are both integral equations with the unknown temperature profile (plug-flow system) or temperature history (batch system) immersed in an exponential term within the integral. The sole experimental variable in the equations developed for determining temperature profiles is the tracer-reaction activation energy. Thus, when using more than one tracer reaction, reactions with different activation energies are needed to gain information. The results are more sensitive to a change in reaction activation energy when the activation energy is low than when it is high. For a given range in activation energies, it is better to use tracer reactions with lower activation energies.

The fundamental relationships are valid for systems both with and without temperature extrema, but the equations with temperature extrema can have multiple solutions. Information additional to the conversion of chemically reacting tracers is required to choose which of the multiple solutions represents the true temperature profile or history of the system. Even without such additional information, important characteristics such as temperature hot spots can be deduced from the results.

Under some circumstances, conversion of a single chemically reacting tracer gives sufficient information for practical purposes. One such situation occurs when the temperature profile can be approximated by a step function. Usually, though, more than one tracer must be used, and the temperature profile or history is obtained by combining the conversions and the fundamental relationship.

Three methods transform the fundamental integral relationships to Fredholm integral equations of the first kind (FIEFKs). The technique of regularization can solve the resulting first-kind Fredholm equations to give reasonably accurate approximations to the true temperature profile or history.

The first of these methods changes the variable from either fractional volume (flow system) or dimensionless time (batch system) to temperature, and employs a temperature distribution function. Using this device enables the fundamental relationship to be converted to a FIEFK. In the second method, the same change of variable is used, but a cumulative distribution function is employed. Again, using this second distribution function can convert the fundamental relationship to a FIEFK. The third method 
expands the fundamental relationship's exponential expression in a Taylor series. It then uses the expansion to create a FIEFK whose unknown distribution is a correction to a previously calculated or assumed temperature history or profile. This leads to an iterative procedure starting with an assumed profile and repetitively improving it until no changes occur.

A fourth approach to solving the fundamental relationship for the temperature history or profile, the optimization of undetermined parameters, also can be used. This is a mature technique and easily used, but suffers from the disadvantage that the expression containing the undetermined parameters predetermines some aspects of the solution.

The three methods involving the use of Fredholm equations were tested for their practicality in obtaining temperature profiles and histories. The technique of regularization can be used to solve the FIEFKs developed in this work. In the first two methods, choice of the optimal value of the regularization smoothing parameter is critical, in common with most applications of this method. A simple criterion for choosing the optimal value of the smoothing parameter seems to work well in most situations studied.

In the third method, the iterative one, the choice of the regularization smoothing parameter is not critical if the parameter is sufficiently large to damp out spurious oscillations.

The temperature profiles obtained using the methods developed here are not particularly sensitive to error in the data. Little difference was detected in the profiles obtained from data containing $0-5 \%$ rootmean-square (rms) error in the data. Reasonable profiles could even be obtained from data containing as much as $10 \%$ rms error in the data.

In a comparison of the three methods, the second and third methods gave solutions of comparable quality. The second method still possesses the difficulty of determining the optimal value of the regularization smoothing parameter. The first method on occasion gave results significantly inferior to those obtained using Methods 2 and 3.

For two types of monotonic temperature profiles examined in this work, an adequate temperature profile may be extracted using as few as two reacting tracers. This approach can distinguish between the two models with as much as $10 \% \mathrm{rms}$ error in the data.

Experimental work has involved two classes of reactions: ester hydrolyses and hydrolyses of bromobenzene derivatives. The former are appropriate as reactive tracers for $\mathrm{HDR}$ reservoirs in the $75-100^{\circ} \mathrm{C}$ temperature range, and the latter in the $150-275^{\circ} \mathrm{C}$ range.

\section{INTRODUCTION}

\section{A. Need for Indirect Measurements of Temperatures within Flowing Systems}

Knowledge of a system's internal temperature characteristics is often necessary for analysis of the system or for prediction of its future behavior. Direct measurement of the temperatures at sufficient locations to characterize the temperature field well enough for analysis or behavior prediction is frequently 
impractical or infeasible. This report presents the mathematical bases for a proposed method for the indirect determination of temperature profiles within flowing systems. The same approach can also determine temperature histories within batch systems.

Early stages in the development of this concept have been reported (Brown et al. 1984; Robinson et al. 1984; 1988). This report brings the development to the point of practical use and explains how the ideas may be implemented.

Flowing systems have fluids continuously transported from points to points within the systems. Many flowing systems have their behavior strongly influenced by the temperature field within them. Examples of systems in which the internal temperature fields are important are continuous chemical reactors, nuclear-waste storage sites, and groundwater systems transporting pollutants. Geothermal reservoirs, whether the HDR, natural steam, or natural hot water type, are flowing systems that need their internal temperatures measured to predict their life. No proven indirect method presently exists for measuring the internal temperature fields of flowing systems. Direct methods for characterizing a temperature field, such as insertion of thermocouples at many locations within the system, are occasionally used. Yet direct methods are usually expensive and often infeasible, and are usually restricted to laboratory investigations (e.g., Smith 1970). Since often there is no practical direct method for measuring temperatures within a system, the development of an indirect technique would be a significant contribution to technology.

\section{B. Need for Temperature Histories of Batch Systems}

The temperature-time relationship in a laboratory batch reactor is extremely desirable for kinetic mechanism studies. A changing temperature adds information that is not available in the usual isothermal batch reactor studies (e.g., Koch 1977). Direct measurement of the temperature history in a batch reactor is normally not as difficult as directly measuring the temperature profile in a flowing system.

Thermocouples or similar devices usually measure temperatures in this type of system. Yet occasions can arise where direct temperature measurement is either difficult or infeasible, and it is for these situations that remote temperature measurement techniques are developed here.

\section{Need for Indirect Temperature Measurements in Hot Dry Rock Geothermal Reservoirs}

The HDR geothermal reservoir process is a technique for extracting the heat from a zone of fractured rock by circulating water through the rock mass. In its simplest form, this heat extraction method involves setting up a steady-state circulation system in which cold water is first pumped into an injection well. It is then forced to bathe a large volume of rock to extract the heat. The process then recovers the injected water as hot, pressurized fluid from a second well. One key issue in the development of HDR technology is the length of time during which a reservoir can produce high-temperature water. As heat extraction proceeds, a cold-temperature wave progresses from the injection well to the production well. 
After a length of time, governed by the flow rate, volume of exposed hot rock, and details of the flow field, the cold-temperature wave will reach the production well. This causes a produced-fluid temperature decline and less efficient conversion of the thermal energy to electrical power. Most heat-transfer models suggest that after a period of lessening performance, the production temperature will have declined to such a low value that the system must be abandoned.

Thermal analysis of small, prototype reservoirs has been manageable by measurable produced-fluid temperature declines, which appear after only a few months of operation (Dash et al. 1983; Murphy et al. 1981; Nicol and Robinson 1990). For larger systems, though, reservoir simulators must be used as predictors because years could pass before a measurable decline in temperature is observed. The normal battery of diagnostic tests (e.g., pressure transient, well logging, conservative tracer, microseismic, and geochemical experiments) does not provide the information necessary to construct detailed reservoir models with the capability of predicting thermal performance. Chemically reacting tracers, which are sensitive to changes in the reservoir's internal temperature field, may provide a usable possibility.

The specific characteristics of the temperature profile between the wells depend on the mean fracture spacing. This causes a variety of possible shapes of the temperature profile (Robinson and Jones 1987). Thus, besides estimating the position of the thermal front, it would be desirable to estimate the shape of the temperature profile in more detail. This would enable a more accurate prediction of thermal decline to be made and a greater understanding about the nature of the heat transfer to be acquired. The reacting tracer technique outlined in the present study in principle allows this added information about the temperature profile to be obtained.

\section{The Possibility of Measuring Temperatures Using Chemical Reactions}

Tests using nonreacting, or conservative, tracers routinely characterize flow fields within flowing systems (e.g., Nauman and Buffham 1983; Fogler and Brown 1992a). These tests usually insert either a pulse of nonreacting substance at a point within the system or make a step change in concentration of a nonreacting substance at the insertion point. The tracer is then withdrawn from another point in the system. The change with time of tracer concentration in the effluent can tell much about the flow field between the points of insertion and withdrawal and occasionally outside this region.

Except for minor effects on density, temperature normally has little effect on conservative tracers, so these tracers give no information on temperatures within the system. On the other hand, the rates of most chemical reactions are extremely sensitive to temperature. The usual mass balance for constant-density reactions (that liquid-phase reactions usually approximate) in isothermal batch systems is

$$
\frac{\mathrm{dC}}{\mathrm{dt}}=-\mathrm{kf}\left(\mathrm{C}_{\mathrm{i}}\right)
$$

and the analogous mass balance for isothermal plug-flow systems is

$$
\mathrm{q} \frac{\mathrm{dC}}{\mathrm{dV}}=-\mathrm{kf}\left(\mathrm{C}_{\mathrm{i}}\right)
$$


Both equations have a reaction-rate expression on the right-hand side (RHS) that is the product of a temperature-dependent portion, $k$, and a concentration-dependent portion, $f\left(C_{i}\right)$. The temperaturedependent parameter $\mathrm{k}$ is frequently termed the reaction-rate constant. For many reactions, the rate constant $k$ follows the Arrhenius relationship $k=A e^{-E / R T}$ (Arrhenius 1889) and can vary by several orders of magnitude over temperature ranges encountered in practical systems.

If reacting chemicals are used as tracers, then conversions of the reactants might give temperature information analogous to the flow fields. In the batch system, it would be the temperature history of the system; in the flow system, it would be the temperature field. Because of the extreme temperature sensitivity of chemical reaction rates to temperature, chemical-reaction conversion might be usable as a quantitative indicator of temperatures within a system. This report develops the mathematical bases for methods using this concept.

\section{E. Plug-Flow, Dispersed-Flow, Perfectly Mixed Batch, and Imperfectly Mixed Batch Systems}

The indirect methods of temperature measurement developed in this report can be applied to both batch and flow systems. Plug flow (or piston flow) is an idealized flow, often approximated in practice (Fogler and Brown 1992a), and this is the type of flow considered in this report. The principles developed here can be used directly for systems that approximate plug flow. Other flow networks can be modeled by several plug-flow systems, and the results of this study are also valid for this problem. The methods developed here can also be applied to perfectly mixed batch systems.

Dispersed-flow systems and imperfectly mixed batch systems are more complicated to model than plug-flow systems. The insight given by the present study is essential for the analysis of these more complex systems. The use of reacting tracers in the more complex systems is presently being investigated, and will be treated in future reports.

\section{THE FUNDAMENTAL RELATIONSHIPS}

\section{A. Basic Assumptions for Batch Systems}

The batch system is assumed to be perfectly mixed at all times. In such a system, there are always no spatial gradients, either temperature or concentration. The temperature and all component concentrations are uniform.

The temperature dependence of the chemical reaction-rate constant is assumed to be that proposed by Arrhenius (1889), i.e., $\mathrm{k}=\mathrm{Ae}^{\mathrm{E} / \mathrm{RT}}$. The rate constants of most simple reactions display this temperature dependence (Glasstone 1946). There are some reactions whose rate constants do not display the Arrhenius temperature dependence, such as the gaseous oxidation of nitric oxide below $350 \mathrm{~K}$ (Bodenstein 1935). Nevertheless, a reaction temperature dependence other than the Arrhenius type is usually evidence for complexity of the reaction path (Benson 1960). This report assumes that all tracer reactions are sufficiently simple that their rate constants have Arrhenius temperature dependences. To apply the 
mathematical developments presented here, the constants for tracer reactions must display the Arrhenius temperature dependence. If they exhibit different types of functional dependence on temperature, the mathematical development must be carried out for the temperature dependences of the reaction constants in question.

It is assumed that the system density does not change significantly throughout the time being studied, which means that the system volume does not change significantly. Two situations can be considered here: one in which the system has large quantities of several different reactants, and the other in which small amounts of reacting tracers are used. In the latter case, the reacting tracers are only a very small fraction of the system, and any density changes caused by the chemical reaction of the tracers should have a negligible effect on the system density. The effect of changing temperature on the physical characteristics of the system is assumed to be insignificant throughout the period being considered.

\section{B. Derivation of the Fundamental Relationship for Batch Systems}

In a constant-density batch system, the usual ordinary differential equation describing a chemical reaction of substance " $\mathrm{i}$ " is

$$
\frac{d C_{i}}{d t}=-k_{i}\left[f\left(C_{i}\right)\right]=-\left(A_{i} e^{-E_{i} / R T}\right)\left[f\left(C_{i}\right)\right]
$$

This applies to the reacting tracer throughout the system. The temperature $T$ depends on the time $t$, i.e., $T=T(t)$. Integrating this over the period being investigated $t_{p}$ yields

$$
\int_{C_{i 0}}^{C_{i r}}\left(\frac{1}{f\left(C_{i}\right)}\right) d C_{i}=-A_{i} \int_{0}^{t_{p}} e^{-E_{i} / R T(t)} d t,
$$

in which $T$ is still a function of $t$. Defining $\xi \equiv t / t_{p}$ as a dimensionless time can allow conversion of Eq. (III-2) to dimensionless form. Changing the limits of the RHS integral appropriately and transferring parameters to the left-hand side (LHS) converts the equation to

$$
-\left(\frac{1}{A_{i} t_{p}}\right) \int_{C_{i 0}}^{C_{i r}}\left(\frac{1}{f\left(C_{i}\right)}\right) d C_{i}=\int_{0}^{1} e^{-E_{i} / R T(\xi)} d \xi \text {. }
$$

In this equation, $\mathrm{T}$ depends on $\xi$. The LHS is a dimensionless group. This group is equivalent to the system average of the reaction-rate constant divided by the rate constant that would exist if the temperature were infinitely high throughout the time of interest. Equation (III-3) is the basic equation for determining temperature-history characteristics of a batch system.

\section{Basic Assumptions for Flowing Systems}

The flow pattern in the first type of flowing system to be considered is plug flow or piston flow. This is the simplest type of flow system, and the principles of the proposed method must be developed for this type first. The flow pattern in many real systems approximates plug flow (Fogler and Brown 1992a), 
and the principles developed here can be used directly for these systems. Other flow networks can be modeled by several plug-flow systems (Fogler and Brown 1992b), and the principles of this report also can be applied to these. Not all flow systems exhibit plug-flow behavior; future reports will treat studies of chemically reacting tracers in non-plug-flow systems.

Again, all tracer reaction-rate constants have the Arrhenius temperature dependence. Section III.A discussed this.

A constant density is also assumed for all flowing systems considered in this report. Analogous to the batch systems, the reacting tracers probably will be only a very small fraction of the flowing liquid. Thus any density changes caused by the chemical reaction of the tracers should have a negligible effect on the density of the flowing liquid.

D. Derivation of the Fundamental Relationship for Flowing Systems

A segment of fluid travels along a particular flow path and encounters different temperatures along the path. A chemically reactive tracer, component " $i$," is added to this segment at a point. In a constantdensity plug-flow system, the usual ordinary differential equation for a chemical reaction is

$$
q \frac{d C_{i}}{d V}=-k_{i}\left[f\left(C_{i}\right)\right]=-\left(A_{i} e^{-E_{i} / R T}\right)\left[f\left(C_{i}\right)\right]
$$

This applies to the reacting tracer in the segment of fluid. The temperature $T$ depends on the fluid segment's position in the flow path, i.e., $T$ is a function of $V$ or $T=T(V)$. The volume traversed by the segment of fluid while traveling in plug flow between the injection and withdrawal points defines the system volume $\mathrm{V}_{\mathrm{s}}$. Integrating Eq. (III-4) over the system volume yields

$$
q \int_{C_{i 0}}^{C_{i r}}\left(\frac{1}{f\left(C_{i}\right.}\right) d C_{i}=-A_{i} \int_{0}^{V_{i}} e^{-E_{i} / R T(V)} d V,
$$

in which $\mathrm{T}$ still depends on V. Defining $\xi \equiv \mathrm{V} / \mathrm{V}_{\mathrm{s}}$ as the system's dimensionless volume allows conversion of Eq. (III-5) to dimensionless form. Changing the limits of the RHS integral appropriately and transferring parameters to the LHS converts Eq. (III-5) to

$$
-\left(\frac{q}{A V_{s}}\right) \int_{C_{i 0}}^{C_{i r}}\left(\frac{1}{f\left(C_{i}\right)}\right) d C_{i}=\int_{0}^{1} e^{-E_{i} / R T(\xi)} d \xi .
$$

Defining the residence time or space time of the system $\tau_{s} \equiv q / V_{s}$ and substituting this into Eq. (III-6) results in

$$
-\left(\frac{1}{A \tau_{s}}\right) \int_{C_{i 0}}^{C_{i r}}\left(\frac{1}{f\left(C_{i}\right)}\right) d C_{i}=\int_{0}^{1} e^{-E_{i} / R T(\xi)} d \xi .
$$

In this equation, $\mathrm{T}$ depends on $\xi$. The LHS of this equation is a dimensionless group, as in Eq. (III-3). Here the group is equivalent to the system average of the reaction-rate constant divided by the 
rate constant that would exist if the temperature were infinitely high throughout the system. Equation (III-7) is the basic equation for determining temperature characteristics of flowing systems.

\section{E. Implications of the Fundamental Relationships}

1. Identical Nature of the Two Fundamental Relationships. Both Eqs. (III-3) and (III-7) have the same form. The difference between the two equations is the time term on the LHSs of the two equations. In the equation derived for batch systems, the time term is the period for which the temperature history is desired. In the equation for flow systems, the term is the residence time or space time that elapses while the segment of fluid containing the tracers travels from the injection point to the withdrawal point. Both time terms represent known quantities of the system.

Since the equations for both batch and plug-flow systems are formally identical, a method suitable for solving the equation for a batch system would be suitable for solving the equation for a plug-flow system. Thus a method found suitable for determining the temperature history of a batch system would also be appropriate for determining the temperature profile of a plug-flow system and vice versa.

2. The Experimental Variable for Multi-Reaction Tracer Tests. The temperature history of the batch, or the temperature profile of the path traversed by the segment of fluid, is $T(\xi)$. Solving Eq. (III-3) or (III-7) for $\mathrm{T}(\xi)$ will give important information about the temperature history or field. In most cases, this will probably require more than one data point, i.e., more than one tracer reaction will be needed. When more than one reaction is necessary, Eqs. (III-3) and (III-7) show that the reaction activation energy must be different for the different reactions. If the E's of the different reactions are all the same, the integral on the RHS of Eq. (III-3) or (III-7) will be identical for all the reactions. This means that the LHS of Eq. (III-3) or (III-7) will be the same for different reactions with the same activation energy. The conversion will merely change to the degree required to keep the equations' LHS constant. No information about the effect of $T(\xi)$ inside the integral will have been obtained beyond that supplied by running one reaction. Only if the reactions have different activation energies will the values of the RHS's integral change. In other words, the reaction activation energy $E$ is the only possible experimental variable for multiple-reaction tracer tests seeking a batch system's temperature history or a flow system's temperature profile.

3. Suitable Classes of Reactions for Reacting Tracer Tests. Equations (III-3) and (III-7) show a limitation on the reaction types that make satisfactory tracer reactions. For these equations to be solvable for $T(\xi)$, the LHS must be obtained by integrating $\left[1 / f\left(C_{i}\right)\right]$ between the limits of the initial and final concentrations. To do this analytically or numerically, there must be no temperature-dependent terms in $f\left(C_{i}\right)$.

Typical forms of $f\left(C_{i}\right)$ are 


$$
\begin{aligned}
& f\left(C_{i}\right)=C_{i}^{n} \quad\left(\text { a type of } n^{\text {th }}\right. \text { order reaction); and } \\
& f\left(C_{i}\right)=\frac{C_{i}}{1+\sum K_{j} C_{j}} \quad \text { (a type of Langmuir-Hinshelwood reaction). }
\end{aligned}
$$

Temperature-sensitive parameters within the $f\left(C_{i}\right)$, such as the $K_{j}^{\prime}$ 's in Eq. (III-9), frequently display an Arrhenius type of exponential temperature dependence.

In the $n^{\text {th }}$ order reactions, there are no temperature-dependent parameters in $f\left(C_{i}\right)$, but there are such parameters in the Langmuir-Hinshelwood rate expressions. With an $n^{\text {th }}$ order reaction, the LHSs of Eqs. (III-3) and (III-7) may be integrated analytically between the limits of the initial and final tracer concentrations. On the other hand, with a Langmuir-Hinshelwood reaction, the LHS cannot be integrated without a costly trial-and-error procedure because of the temperature-dependent terms within it. Thus an $\mathrm{n}^{\text {th }}$ order reaction might nake a good tracer reaction, while a Langmuir-Hinshelwood reaction probably would not.

\section{Pulse and Continuous Injection of Reacting Tracers into Flowing Systems. When} conservative (i.e., nonreacting) tracers characterize flow patterns, the unsteady-state behavior of these tracers gives the information. Pulse injections or negative or positive steps of the tracer input are the usual manners of creating unsteady tracer inputs (Fogler and Brown 1992a). Sine-wave inputs have also been considered (Wen and Fan 1975). Yet for reacting tracers, the unsteady state is not necessarily a factor in the analysis. Equations (III-4)-(III-7) are valid for both injection of a pulse and continuous flow of reacting tracers. This is true because of the plug flow of the fluid. The analysis of both pulse injection and continuous flow of reacting tracers is therefore identical.

In dispersed flow, continuous flow of reacting tracers causes tracer behavior different from that which occurs when tracer pulses are injected. This will be the subject of future studies.

\section{F. Use of First-Order Reactions}

The rest of this report will use first-order reactions, i.e., $f\left(C_{i}\right)=C_{1}$. As stated above (Section III.E.3), other types of reactions can be used as temperature tracers. Derivation of the appropriate equations for these other reactions follows directly by integrating the LHS of either Eq. (III-3) or (III-7).

For a first-order reaction, integration of the LHS of Eq. (III-3) gives

$$
-\left(\frac{1}{A_{i} t_{p}}\right) \ln \left(\frac{C_{i f}}{C_{i 0}}\right)=\int_{0}^{1} \mathrm{e}^{-E_{i} / R T(\xi)} \mathrm{d} \xi \quad .
$$

Inserting several tracers with different reaction activation energies $E_{i}$ and different pre-exponential factors $A_{i}$ into the batch system at $t=0$ causes Eq. (III-10) to become

$$
-\left(\frac{1}{A_{i} t_{p}}\right) \ln \left(\frac{C_{i f}}{C_{i 0}}\right)=\int_{0}^{1} e^{-E_{i} / R T(\xi)} d \xi \quad i=1,2, \ldots, n .
$$


The analogous equation for using several tracers in a plug-flow system is

$$
-\left(\frac{q}{A_{i} V_{s}}\right) \ln \left(\frac{C_{i f}}{C_{i 0}}\right)=\int_{0}^{1} e^{-E_{i} / R T(\xi)} d \xi \quad i=1,2, \ldots, n,
$$

or

$$
-\left(\frac{1}{A_{i} \tau_{s}}\right) \ln \left(\frac{C_{i r}}{C_{i 0}}\right)=\int_{0}^{1} e^{-E_{i} / R T(\xi)} d \xi \quad i=1,2, \ldots, n
$$

The LHS of Eq. (III-11) or (III-13) can be calculated for each tracer from the initial and final concentrations. Let $g\left(E_{i}\right)=-\left(1 / A_{i} t_{p}\right)\left[\ln \left(C_{i r} / C_{i 0}\right)\right]$ or $g\left(E_{i}\right)=-\left(1 / A_{i} \tau_{s}\right)\left[\ln \left(C_{i t} / C_{i 0}\right)\right]$, whichever is appropriate, and

$$
g\left(E_{i}\right)=\int_{T_{\min }}^{T_{\max }} e^{-E_{i} / R T(\xi)} d \xi \quad i=1,2, \ldots, n
$$

To calculate $T(\xi)$ from the observed $g\left(E_{i}\right)$ 's, the integral equation (III-14) must be inverted to obtain the function $T(\xi)$.

\section{SINGLE-REACTION TRACER TESTS}

Single-reaction experiments are simple to carry out in principle, and they can give important information about the temperature characteristics of flowing systems. On occasion, the temperature information so obtained may be sufficient and a multiple-reaction tracer test superfluous. This would occur when the form of the temperature distribution or history is known, and only the value of a single parameter in the distribution is needed. For example, in the thermal drawdown of a HDR geothermal reservoir that can be modeled as a single rectangular fracture, the axial temperature profile within the fracture has been reasoned to follow the relationship

$$
\frac{T-T_{0}}{T_{r}-T_{0}}=\left(\frac{\lambda_{r} A_{r}}{\rho c_{p} q \sqrt{a_{r} t_{B}}}\right) \xi \quad \text { (Murphy et al. 1981). }
$$

In this equation, the terms inside the brackets can be lumped together as a constant times the fractional volume (or fractional distance $\xi$ along the fracture). This constant may be found by numerical solution of Eq. (III-7) from $\xi=0$ to $\xi=1$ for different values of $\lambda_{\mathrm{r}} A_{\mathrm{f}} / \rho c_{\mathrm{p}} q \sqrt{\mathrm{a}_{\mathrm{r}} \mathrm{t}_{\mathrm{g}}}$ to obtain the conversion of reacting tracer as a function of this parameter. The conversion of the tracer will then give the value of the dimensionless constant $\lambda_{\mathrm{r}} A_{\mathrm{f}} / \rho c_{\mathrm{p}} q \sqrt{a_{\mathrm{r}} t_{\mathrm{g}}}$, which in turn can be used to calculate the temperature profile in the fracture.

Analogous work could be carried out for other circumstances. Any time there is a temperature history or profile characterized by one parameter, it is possible that a single-reaction tracer test can determine the value of this parameter. 


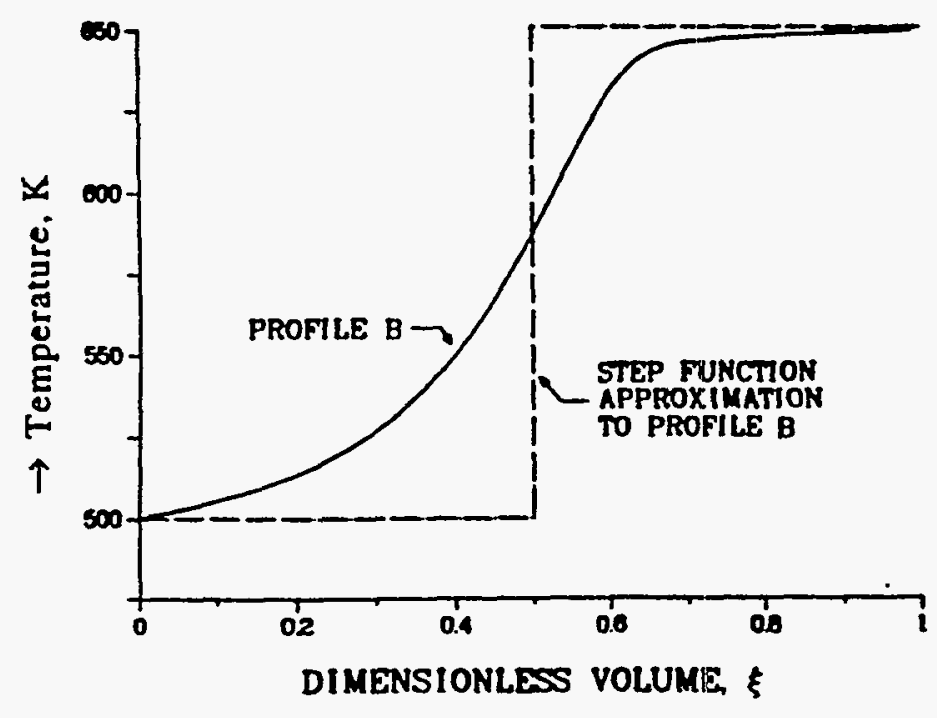

Figure 1. Step-function approximation to an S-shaped temperature profile.

The simplest one-parameter temperature profile is the step function. Sometimes other profiles can be approximated by a step, and this may be sufficient for a particular situation. Figure 1 shows an $\mathrm{S}$-shaped profile approximated in this fashion. (This is the Profile $\mathrm{B}$, characteristic of a type of HDR reservoir with about half its potential energy extracted. This is one of the profiles used later in the report for evaluating different approaches to extracting temperature patterns from multiple-reaction tracer data.) Assuming a step temperature profile and negligible conversion at the lower temperature, integration of the RHS of Eq. (III-12) is trivial and the equation becomes simply

$$
-\left(\frac{q}{A_{1} V_{s}}\right) \ln \left(\frac{C_{1 f}}{C_{10}}\right)=\left[e^{-E_{1} / R T_{\max }}\right]\left(\xi_{1}\right) \text {. }
$$

In this situation, $T_{\max }$ is the temperature of the upper portion of the step, and $\xi_{1}$ the fraction of $V_{s}$ at the higher temperature. The parameter $\xi_{1}$ normally would be the unknown for which this equation would be solved. The negligible nature of the conversion at the lower temperature should be true for significant changes in temperature and typical activation energies. For example, if $\mathrm{T}_{\min }=200^{\circ} \mathrm{C}, \mathrm{T}_{\max }=300^{\circ} \mathrm{C}$, $E=130 \mathrm{~kJ} / \mathrm{mol}$, and $\xi_{1}=0.5$, the error in $\xi_{1}$ from ignoring the reaction in the cooler region is only $0.3 \%$.

\section{EQUATIONS FOR MULTIPLE-REACTION TRACER TESTS OF MONOTONIC SYSTEMS}

\section{A. Forms of Equations Considered}

1. Equations for Batch and Plug-Flow Systems. Section III.E. I stated that the equations for obtaining the temperature history of batch systems and for obtaining the temperature profiles of plug-flow 
systems are formally identical. The methods developed for extracting the solution for one type of system can therefore be used for the other. Since treating each system separately would be redundant, the next sections of this report consider principally the problem of obtaining temperature profiles in plug-flow systems. The methods developed for this type of system are directly applicable to obtaining the temperature histories of batch systems.

2. Fredholm Integral Equations of the First Kind. A single-reaction tracer test may on occasion give information adequate for a situation. Usually, however, the internal temperature characteristics of a system are sufficiently complex that even minimal description of the temperature field requires more than one piece of information. Then, $n>1$ in Eq. (III-14), and several tracer reactions must be carried out either separately or simultaneously.

To obtain the temperature profile in a flowing system from the conversion of several reacting tracers, Eq. (III-14) must be inverted to obtain $T(\xi)$. There are some difficulties inherent in inverting Eq. (III-14). The equation involves a nonlinear term, $\exp \left[-E_{\mathrm{j}} / \mathrm{RT}(\xi)\right]$, and the unknown function $\mathrm{T}(\xi)$ is embedded within this term. While some methods can treat Eq. (III-14) in this form, most treatments of integral equations consider the form

$$
g(x)=\int_{a}^{b} K(x, y) f(y) d y .
$$

If $a$ and $b$ are constants, this is a Fredholm integral equation of the first kind (FIEFK). A large amount of prior work exists on the treatment and inversion of this type of equation (e.g., Miller 1974, Allison 1979, Wing 1984). To make use of this work, Eq. (III-14) must be modified to the form of Eq. (V-1). The next paragraphs present three approaches that modify Eq. (III-14) to give three different equations in the form of Eq. (V-1). There is also a brief discussion of the parameter-optimization approach to solving particular applications of Eq. (III-14).

\section{B. A Fredholm Equation Using a Simple Temperature-Distribution Function}

A temperature-distribution function $\eta(T)$ is defined so that $\eta(T) \delta T$ is the fraction of the system volume having temperatures between $T$ and $T+\delta T$. Some flow systems have monotonic temperature profiles, i.e., the temperature rises or falls continuously throughout the length of the system. For systems with monotonic profiles, this distribution function may be used to eliminate $\xi$ from Eq. (III-14). The definition of $\eta(T)$ says that

$$
\delta V=V_{s} \eta(T)|\delta T| \text {, or }\left|\frac{d V}{d T}\right|=V_{s} \eta(T) \text {, or }\left|\frac{d \xi}{d T}\right|=\eta(T)
$$

Thus $\eta(T)$ is the inverse of the slope of the temperature profile, $d T / d \xi$. Where the temperature profile is flat, $\eta(T)$ assumes a large value. Where the temperature profile is steep, $\eta(T)$ is small. Using $\eta(T), \xi$ can be eliminated from Eq. (III-14), yielding 


$$
g\left(E_{i}\right)=\int_{T_{\min }}^{T_{\max }} e^{-E_{i} / R T} \eta(T) d T \quad i=1,2, \ldots, n
$$

Equation (V-3) is a FIEFK. It is the first of the four principal equations studied in this report for determining temperature characteristics using chemically reacting tracers.

\section{A Fredholm Equation with a Cumulative Temperature-Distribution Function}

Another distribution function is the fraction of the system volume at a temperature $T$ or less:

$$
H(T) \equiv \int_{T_{\min }}^{T} \eta(s) d s=\text { the fraction of the system volume at a temperature } T \text { or less. }
$$

In this definition, the letter $s$ is a dummy variable. This distribution function appears if the integral on the RHS of Eq. $(\mathrm{V}-3)$ is integrated by parts:

$$
\int_{T_{\min }}^{T_{\max }}\left(e^{-E_{i} / R T}\right) \eta(T) d T=\left\{\left(e^{-E_{i} / R T}\right)\left[\int_{T_{\min }}^{T} \eta(s) d s\right]\right\} \mid T_{\max }-\int_{T_{\min }}^{T_{\max }}\left[\int_{T_{\min }}^{T} \eta(s) d s\right]\left(e^{-E_{i} / R T}\right)\left(\frac{E_{i}}{R T^{2}}\right) d T .
$$

Because

$$
\int_{T_{\min }}^{T_{\max }} \eta(T) d t=1
$$

Eqs. (V-3), (V-5), and (V-6) can be combined to give

$$
e^{-E_{i} / R T_{\max }}-g\left(E_{i}\right)=\int_{T_{\min }}^{T_{\max }} \frac{E_{i}}{R T^{2}} e^{-E_{j} / R T} H(T) d T \quad i=1,2, \ldots, n
$$

Equation (V-7) is also a FIEFK. It is another of the four principal equations for determining internal temperature characteristics using chemically reacting tracers that this report studies.

For monotonically increasing or decreasing temperature profiles, $H(T)$ is the functional inverse of $T(\xi)$, i.e., $H[T(\xi)]=\xi$. For naturally occurring temperature profiles, $H(T)$ can be expected to be a smooth function, whereas $\eta(T)\left(=|\mathrm{d} T / \mathrm{d} \xi|^{-1}\right)$ exhibits a sharp peak whenever the temperature profile possesses a flat region.

\section{A Fredholm Equation Containing a Series Expansion Leading to an Iterative Solution}

There may be an initial estimate of the temperature profile. If so, then an approximate difference between the estimate and the true solution can be developed and incorporated into an iterative method for obtaining a temperature profile. The initial estimate is $L_{0}(\xi)$, and the fractional deviation of $L_{0}(\xi)$ from the true profile $T(\xi)$ is

$$
\varepsilon(\xi) \equiv \frac{T(\xi)-L_{0}(\xi)}{L_{0}(\xi)}
$$


The nonlinear term $\exp \left[-\mathrm{E}_{\mathrm{i}} / \mathrm{RT}(\xi)\right]$ in Eq. (III-14) can be expanded in terms of $\mathrm{L}_{0}(\xi)$ and $\varepsilon(\xi)$ :

$$
\exp \left[\frac{-E_{i}}{R T(\xi)}\right]=\left\{\exp \left[\frac{-E_{i}}{R L_{0}(\xi)}\right]\right\}^{\{1 /[1+\varepsilon(\xi)]\}} \approx\left\{\exp \left[\frac{-E_{i}}{R L_{0}(\xi)}\right]\right\}^{[1-\varepsilon(\xi)]} .
$$

Expanding the RHS of Eq. (V-9) in a Taylor series, and neglecting all terms of degree equal to or higher than $\varepsilon^{2}(\xi)$, yields

$$
\exp \left[\frac{-E_{i}}{R T(\xi)}\right] \approx\left\{\exp \left[\frac{-E_{i}}{R_{0}(\xi)}\right]\right\}\left[1+\frac{\varepsilon(\xi) E_{i}}{R L_{0}(\xi)}\right] .
$$

Substitution of Eq. (V-10) into Eq. (III-14) gives a FIEFK:

$$
g\left(E_{i}\right)-\int_{0}^{1} \exp \left(\frac{E_{1}}{R L_{0}(\xi)}\right) d \xi=\int_{0}^{1}\left(\frac{E_{i}}{R L_{0}(\xi)}\right)\left[\exp \left(\frac{E_{i}}{R L_{0}(\xi)}\right)\right] \varepsilon(\xi) d \xi \quad i=1,2, \ldots, n .
$$

Solution of Eq. $(V-11)$ obtains $\varepsilon(\xi)$ for an assumed temperature profile $L_{0}(\xi)$. Since $\varepsilon(\xi)$ is a fractional deviation of $T(\xi)$ from $L_{0}(\xi)$, the estimate of the temperature profile $L_{0}(\xi)$ can be modified to give an improved estimate. The improved estimate becomes the new $L_{0}(\xi)$ and a new solution of Eq. (V-11) obtains a new $\varepsilon(\xi)$. This procedure can be iterated, and iteration $k+1$ obtains the new assumed profile from

$$
\mathrm{L}_{0}{ }^{k+1}(\xi)=\mathrm{L}_{0}{ }^{k}(\xi)\left[1+\varepsilon^{k}(\xi)\right]
$$

In all situations we have tested thus far, $\varepsilon(\xi)$ has converged to very small values; thus this iterative procedure can continue until the L-2 norm of $\varepsilon(\xi)$ becomes sufficiently small. The usual determining factor in choosing the desired L-2 norm of $\varepsilon(\xi)$ is the level of error in the data.

\section{E. An Equation Used for Parameter Optimization}

1. Function to be Minimized. A commonly used approach to solving integral equations for an unknown functional relationship involves parameter optimization. If one is seeking the temperature profile in a plug-flow system or temperature history in a batch system, the gross characteristics of the profile shape are sometimes known. When this situation exists, postulation of an analytic function with undetermined parameters $\beta_{1}, \beta_{2}, . . \beta_{j}, . ., \beta_{m}$ that also possesses the profile characteristics can lead to a solution. Evaluation of these undetermined parameters within the postulated profile function gives the actual temperature profile.

Let the postulated profile be $T\left(\xi, \beta_{1}, \beta_{2}, . . \beta_{j}, . ., \beta_{m}\right)$. Substituting this for $T$ in Eq (III-14) yields

$$
g\left(E_{i}\right)=\int_{0}^{1} e^{-E_{i} / R T\left(\xi, \beta_{1}, \beta_{2}, \ldots \beta_{j}, \ldots \beta_{m}\right)} d \xi
$$


Equation (V-13) contains the postulated profile $T\left(\xi, \beta_{1}, \beta_{2}, . . \beta_{\mathrm{j}}, . ., \beta_{\mathrm{m}}\right)$. This means a prejudgment exists concerning the general characteristics of the system's temperature profile. If this judgment is incorrect, then the profile resulting from inverting Eq. (V-13) will probably be incorrect.

Thus, this approach involves choice of a type of temperature profile, then postulation of a functional form describing this type. The function has the adjustable parameters $\beta_{1}, \beta_{2}, . . \beta_{j}, . ., \beta_{m}$. Then this process creates an objective function $\phi$ whose minimization yields the "best" values of the adjustable parameters. One such objective function that might be minimized by adjusting the parameters to their optimal values is

$$
\phi=\sum_{i}\left[g\left(E_{i}\right)-\int_{0}^{1}\left[e^{-E_{i} / R T\left(\xi_{1}, \beta_{1}, \beta_{2}, \ldots \beta_{j} \ldots, \beta_{m}\right)}\right] d \xi\right]^{2} .
$$

Yet this function would give too much emphasis to low-activation-energy data because of the strong effect of the exponential term. An objective function that eliminates this emphasis, whose minimization gives equal weight to each data point, is

$$
\phi=\sum_{i}\left[1-\frac{\int_{0}^{1}\left[\mathrm{e}^{-E_{i} / R T\left(\xi, \beta_{1}, \beta_{2}, \ldots \beta_{j} \ldots, \beta_{m}\right)}\right] d \xi}{g\left(E_{i}\right)}\right]^{2} .
$$

2. The Optimization Procedure. Minimization of a function by optimizing several undetermined parameters is a mature technique covered in many texts (e.g., Edgar and Himmelblau 1988; Riggs 1988). The techniques used are invariably numerical, and the most popular methods are usually based on the Gauss-Newton method or some variation or extension of this approach (cf. Gill 1981).

The two standards for judging the validity of the temperature function obtained by this approach are those common to most parameter optimizations. The first standard is a variance that is about the same as the error in the data. The second is the absence of discernible trends when comparing the behavior of the data with that of the RHS of Eq. (V-13) as the activation energy changes.

For the greatest precision in an adjustable parameter, the variable $E$ should be chosen to maximize the second derivative of $\phi$ with respect to that parameter, i.e., the experimental $E_{i}$ 's should maximize the absolute value of $\partial^{2} \phi / \partial \beta_{i}^{2}$. Appendix A presents the proof that maximizing the value of this second derivative gives the greatest precision of the adjustable parameter.

\section{EQUATIONS FOR SYSTEMS CONTAINING TEMPERATURE EXTREMA}

\section{A. The Equations for Monotonic Systems}

In Section $\mathrm{V}$, four equations were derived describing monotonic systems, i.e., systems with temperatures continually increasing or decreasing throughout their lengths. These equations are 


$$
\begin{gathered}
g\left(E_{i}\right)=\int_{T_{\text {min }}}^{T_{\text {max }}} e^{-E_{i} / R T} \eta(T) d T \quad i=1,2, \ldots, n, \\
e^{-E_{i} / R T_{\max }}-g\left(E_{i}\right)=\int_{T_{\min }}^{T_{\max }} \frac{E_{i}}{R T^{2}} e^{-E_{i} / R T} H(T) d T \quad i=1,2, \ldots, n, \\
g\left(E_{i}\right)-\int_{0}^{1} \exp \left(\frac{E_{i}}{R L_{0}(\xi)}\right) d \xi=\int_{0}^{1}\left(\frac{E_{i}}{R L_{0}(\xi)}\right)\left[\exp \left(\frac{E_{i}}{R L_{0}(\xi)}\right)\right] \varepsilon(\xi) d \xi \quad i=1,2, \ldots, n .
\end{gathered}
$$

and

$$
g\left(E_{i}\right)=\int_{0}^{1} e^{-E_{i} / R T\left(\xi, \beta_{1}, \beta_{2}, \ldots, \beta_{m}\right)} d \xi
$$

Two of these equations, Eqs. (V-11) and (V-13), are obviously applicable to systems with temperature extrema. The solution to Eq. (V-13) depends on the form of the solution initially postulated. If a solution form with one or more extrema is postulated, and the system possesses these extrema, the solution will probably possess these extrema.

The solution to Eq. (V-11) gives a correction to a postulated temperature profile. If the postulated profile contains extrema close to the extrema possessed by the system, it is likely the correction will move the calculated profile closer to the true one. The possibility of multiple solutions in this situation is discussed below in Section VI.C.

For two of the equations derived for monotonic systems, Eqs. (V-3) and (V-7), the applications to systems with temperature extrema are not immediately evident. The next paragraphs discuss these applications.

\section{B. Distribution Functions in Systems Containing Temperature Extrema}

Equation (V-3) was derived in Section V.B for monotonic systems. The identical equation can describe systems with temperature extrema. Appendix B shows the derivation of this equation for systems with temperature extrema.

When the system has a monotonic temperature profile, Eq. (V-2) develops the idea that the temperature-distribution function is equal to the absolute value of the slope inverse at a particular temperature, i.e., $\eta(T)=|d \xi(T) / d T|=|d T / d \xi|^{-1}$. The derivation of Eq. (V-3) uses this. Yet in systems where there are temperature extrema, this is not true. The distribution function equals the sum of the slopeinverse absolute values at that temperature, i.e.,

$$
\eta(T)=\Sigma|d \xi(T) / d T|=\Sigma\left(|d T / d \xi|^{-1}\right) .
$$

Equation (V-7) uses a cumulative distribution function $H(T)$, defined by Eq. (V-4) and based on the distribution function $\eta(T)$. Since Eq. (V-3), using the $\eta(T)$ of Eq. (VI-1), applies to systems with any number of extrema, Eq. (V-7) also applies to systems with any number of extrema. 


\section{Multiple Solutions in Systems Containing Temperature Extrema}

Once the simple distribution function $\eta(T)$ is obtained for a monotonic system with known boundary conditions, the temperature profile corresponding to the distribution function is unique. The same is true for the cumulative distribution function $H(T)$. The appropriate calculation yields the temperature profile directly from either distribution function.

In systems with temperature extrema, however, a single distribution function can correspond to multiple temperature profiles. For example, mirror images exhibit identical values of $\Sigma|d \xi(T) / d T|$ at any value of $T$, and thus have identical distribution functions $\eta(T)$ over the entire range of $T$. If the boundary conditions are specified for the problem, the mirror images would also need the same boundary conditions. Such a system is possible. Similarly, other differing temperature profiles can be constructed that still possess identical $\eta(T)$ 's.

Equation (III-14) has $T(\xi)$ as the unknown distribution function. Equation (V-3), with $\eta(T)$ as the unknown distribution function, follows directly from Eq. (III-14). A simple change of variable accomplishes this. As a result, any $T(\xi)$ constructed from the $\eta(T)$ obtained by solving Eq. $(V-3)$ satisfies Eq. (III-14). Since multiple temperature profiles are inherently possible for a single $\eta(T)$ in systems with temperature extrema, multiple solutions to Eq. (III-14) are possible if the system has temperature extrema.

Each simple distribution function $\eta(T)$ has a cumulative distribution function $H(T)$ corresponding to it. Just as multiple temperature profiles can be constructed from any $\eta(T)$ in a system containing temperature extrema, so the same profiles can be constructed from the $H(T)$ corresponding to the particular $\eta(T)$.

Since multiple solutions to Eq. (IIl-14) are possible, the iterative approach to solving Eq. (III-14) via Eq. (V-11) might find multiple solutions. Section VIII.3.E below examines this possibility.

Thus Section B of this chapter concludes that the equations derived for monotonic systems are also valid for systems with temperature extrema. Then Section $C$ shows that whereas there is a single "best" solution to Eq. (III-14) for a monotonic system, multiple solutions of equal quality are possible for a system containing temperature extrema.

\section{SOLUTION PROCEDURES FOR THE FREDHOLM EQUATIONS}

\section{A. Difficulties Inherent in Inverting First-Kind Fredholm Equations}

Direct methods attempting to solve FIEFKs almost invariably give chaotic solutions (Allison 1979). There is a nonuniqueness in the solution if any kind of error exists in Eq. (V-1)'s experimental function $g(x)$, even error as small as computer roundoff. For example, if the system distribution function $f(y)$ in Eq. $(V-1)$ is converted to $f(y)+\gamma \sin \omega y$, Eq. $(V-1)$ becomes 


$$
g(x)=\int_{a}^{b} K(x, y)[f(y)+\gamma \sin \omega y] d y
$$

If there is any error in $\mathrm{g}(\mathrm{x})$, there is always some frequency above which there lies an infinite number of frequencies that gives an infinite number of solutions equal to $f(y)+\gamma \sin \omega y$ satisfying Eq. (V-1). The higher the frequency, the higher $\gamma$ can be, and the more chaotic the solution. It is this inherent "ill-posed" nature of the FIEFKs that makes them so difficult to solve and obtain reasonable answers.

\section{B. A General Method of Regularization That Can Incorporate Constraints and Boundary Conditions.}

One direct approach to solving FIEFK (V-1) involves finding the function $f(y)$ that minimizes locally the integral

$$
I=\int_{x=c}^{x=d}\left[g(x)-\int_{y=a}^{y=b} K(x, y) f(y) d y\right]^{2} d x
$$

Because this is a direct approach to solving the Fredholm equation, it almost invariably gives chaotic solutions as stated in the previous section. One way to resolve this difficulty, known as regularization (Tikhonov and Arsenin 1977), has been the addition of a stabilizing term $\alpha \mathrm{G}(\mathrm{f})$ to the RHS of Eq. (VII-2), i.e.,

$$
I=\int_{x=c}^{x=d}\left[g(x)-\int_{y=a}^{y=b} K(x, y) f(y) d y\right]^{2} d x+\alpha G(f)
$$

The term $G(f)$ is a nonlinear differential operator on $f(y)$ with non-negative coefficients and $\alpha$ is a nonnegative adjustable parameter. If $G(f)$ has an appropriate form [e.g., $G(f)=\int f^{2} d y$ ], the addition of this term smooths the solution for $f(y)$. The value of $\alpha$ depends on the degree of smoothness desired. If $\alpha$ is too large, the solution may be over-smoothed and valuable information lost. If $\alpha$ is too small, spurious structures may appear in the solution. Choosing the proper value of $\alpha$ is critical to successful application of the regularization approach (Miller 1974). Occasionally, individual characteristics of the equation or the problem will suggest the proper value of $\alpha$ (e.g., Bischke et al. 1991). Still, no completely reliable general criterion presently exists for choosing the optimal $\alpha$ (Brown and Travis 1984; v. Szombathely et al. 1992).

For the first two methods in this study, the equations were solved using various values of $\alpha$. The $\alpha$ selected as "the best" or optimal was the one for which the resulting $f(y)$ minimized the integral given by Eq. (VII-2). Although this strategy proved to be effective for Methods 1 and 2, it is not always applicable (Brown and Travis 1984). (An isolated case, when the strategy did not work, is mentioned later in this study.) 
This study employed a first-order stabilizer, given by Eq. (VII-4):

$$
G(f)=\int_{a}^{b}\left\{q_{0}[f(y)]^{2}+q_{1}[d f(y) / d y]^{2}\right\} d y
$$

In general regularization, once the functional form of $G(f)$ is chosen, calculus of variations can obtain the necessary condition to minimize the RHS of Eq. (VII-3) (see Britten et al. 1983, for details). Converting $\mathrm{g}(\mathrm{x})$ to a discrete form as an $\mathrm{n}$-valued vector and establishing a grid of $\mathrm{m}$ mesh points (i.e., $\mathrm{m}$ divisions in the interval $y[a, b])$ can transform the necessary condition into a matrix equation of the form

$$
[\mathbf{M}+\alpha \underline{H}] \underline{\mathbf{f}}=\underline{\mathbf{h}} \text {. }
$$

The vector $\mathbf{f}$ denotes the discrete form of function $\mathrm{f}(\mathrm{y})$. Appendix $\mathrm{C}$ lists the expressions for $\mathbf{M}, \mathbf{H}$ (both $\mathrm{m} \times \mathrm{m}$ matrices), and $\underline{\mathrm{h}}$ for various situations.

Changing the relative values of $q_{0}$ and $q_{l}$ can show the effect of the derivative term in Eq. (VII-4) on the solution.

\section{The Butler, Reeds, and Dawson Method Incorporating the Non-Negativity Constraint}

In many physical applications, the distribution function $f(y)$ is non-negative. Butler, Reeds, and Dawson (1981) proposed a way to incorporate the non-negativity constraint while minimizing the objective function for regularization. Instead of directly computing the m-value vector $\mathbf{f}$, their approach computes $\mathbf{f}$ through an n-value vector $\underline{c}$, which is found by an iterative procedure. Britten et al. (1983) reported significant improvement in their results when they incorporated the non-negativity constraint by using the approach of Butler et al. (the BRD approach). This technique requires the inversion of $\mathrm{n} \times \mathrm{n}$ matrices, instead of $\mathrm{m} \mathrm{x} \mathrm{m}$ matrices in the traditional regularization scheme. In the usual situation, the number of mesh points exceeds the number of data points, i.e., $m \gg n$. Here, the BRD method becomes computationally efficient. Yet their approach has two drawbacks. When boundary conditions are known, it might improve the results if they could be incorporated into the algorithm as solution constraints. Since the $\mathrm{BRD}$ computational method is somewhat indirect, it is not obvious how to impose the boundary conditions on $f(y)$. In addition, Butler et al. (1981) devised their method for only one form of the stabilizing term, namely $G(f)=[f(y)]^{2}$, i.e., $q_{0}=1$ and $q_{1}=0$ in Eq. (VII-4). This is the simplest version of the stabilizing term, the zero-order stabilizer, and enjoys wide use. In situations warranting a higherorder stabilizer, their method cannot be used in its present form.

\section{An Iterative Method That Gives the Smoothest Solution at Any Desired Accuracy}

As mentioned above, choice of the smoothing parameter $\alpha$ is critical to success in most regularization approaches. Section VII.B states that a simple criterion for choosing $\alpha$ was adequate in most situations found in the present study. Still, use of this simple criterion is not always successful 
(Brown and Travis 1984). The iterative approach developed in Section V.C can avoid this difficulty. This iterative approach employs a fixed value of $\alpha$, sufficiently high to avoid spurious peaks in the solution, for all iterations. Numerical experiments have shown that using an $\alpha$ somewhat larger than the optimal value does not degrade the solution appreciably. It is also much safer than risking an $\alpha$ smaller than the optimal value. As explained earlier, $\varepsilon(\xi)$ is computed and $L_{0}(\xi)$ modified accordingly at each iteration.

Although this approach requires more iterations, the strategy is computationally efficient; at each iteration it solves the problem for only one value of $\alpha$. Also, with the use of a sufficiently high value of $\alpha$, this approach significantly decreases the possibility of spurious structures occurring in the result.

\section{TESTING THE EQUATIONS AND SOLUTION PROCEDURES}

\section{A. Three Typical Temperature-Profile Functions}

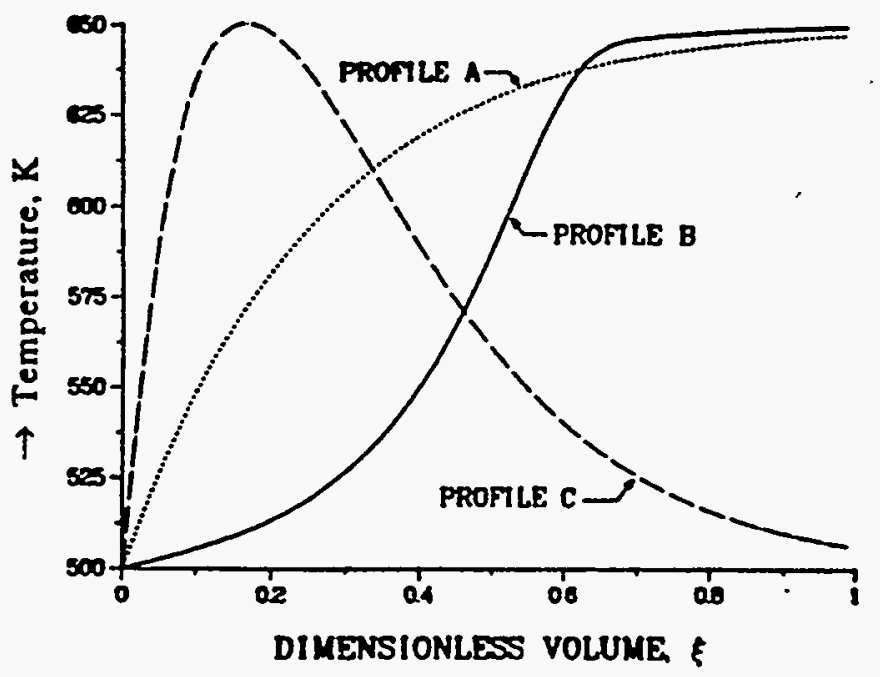

Figure 2. Types of temperature profiles for typical flowing systems. A. Adiabatic reactor with exothermic reaction or Hot Dry Rock reservoir with conduction-limited heat transfer. B. Highly fractured Hot Dry Rock reservoir. C. Plug-flow chemical reactor with exothermic reaction and external heat exchange.

1. Analytic Expressions for Three Temperature-Profile Types. Figure 2 presents three types of temperature profiles. Profile $\mathrm{A}$ has the characteristic shape exhibited by an adiabatic tubular reactor with an exothermic reaction occurring inside it. Profile A also has the characteristic shape exhibited by a type

- HDR geothermal reservoir. Profile B has characteristics of another type of HDR reservoir with about 'ntial thermal energy extracted. Profile $\mathrm{C}$ has the temperature characteristics of a tubular oxothermic reaction is taking place and whose wall is being cooled. The three - following relationships: 


$$
\begin{array}{ll}
\text { Profile A: } & T(\xi)=500+(150)\left[1-\mathrm{e}^{-5.0 \xi}\right] ; \\
\text { Profile B: } & T(\xi)=500+(150)\left\{1-\mathrm{e}^{-[0.5 /(1-\xi))^{+}}\right\}\left(1-\mathrm{e}^{-5 \xi}\right) ; \\
\text { Profile C: } & T(\xi)=500+(150)\left[\beta_{1} \xi \mathrm{e}^{-\beta_{2} \xi}\right] .
\end{array}
$$

The analytical forms of these equations have no theoretical significance; they were chosen to give the profile shapes resulting fiom the physical situations described. The values of the parameters in the equations provide reasonable profiles, each with an entrance temperature of $500 \mathrm{~K}$ and a maximum temperature of approximately $650 \mathrm{~K}$. (The two parameters in Profile $\mathrm{C}$ are used to adjust the location of the maximum temperature. For the early evaluations, $\beta_{1}=16.31$ and $\beta_{2}=6.00$, locating $T_{\max }$ at $\xi=0.167$.)

These three temperature profiles are used below to test the numerical methods proposed for inverting Eq. (III-14) to obtain temperature profiles in flowing systems from multireaction tracer tests.

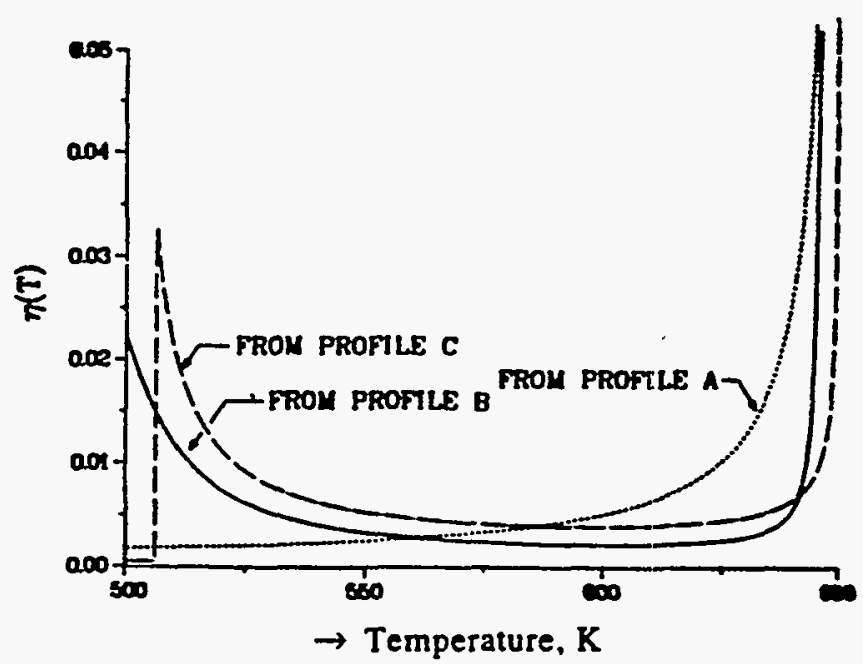

Figure 3. Simple temperature distributions corresponding to profiles in Fig. 2. A. Adiabatic reactor with exothermic reaction or Hot Dry Rock reservoir with conduction-limited heat transfer. B. Highly fractured Hot Dry Rock reservoir. C. Plug-flow chemical reactor with exothermic reaction and external heat exchange.

\section{The Simple Temperature Distributions for the Three Typical Temperature Profiles.}

Figure 3 shows the simple temperature distribution functions corresponding to the three temperature profiles of Fig. 2. As discussed in Section V.C, at temperatures where the profiles are flat, the $\eta(T)$ 's become very large because large fractions of the system volumes occur over small temperature ranges. A zero slope in the temperature profile will cause $\eta(T)$ to approach infinity at that temperature. When the slopes of the profiles are high, the $\eta(T)$ 's become small because only small fractions of the system volumes occur at those temperatures. In the distribution function for Profile $\mathrm{B}$, the peak at the high 
temperature reflects the flat portion of the temperature profile at that point. The flattening out of the profile at the lower temperatures causes the distribution function's peak there. Similar correspondences exist for the other two profiles.

3. The Cumulative Temperature Distributions for the Three Typical Temperature Profiles. A cumulative temperature distribution $H(T)$ was defined in Section V.D. Figure 4 shows the $H(T)$ curves corresponding to the three temperature profiles of Fig. 2. For monotonically-increasing profiles like $\mathrm{A}$ and $\mathrm{B}, \mathrm{H}(\mathrm{T})$ has the appearance of the temperature profile with abscissa and ordinate reversed. For profiles with one or more maxima, the cumulative distribution is different from this because for a given temperature, volumes on either side of the various maxima must be added to obtain $H(T)$.

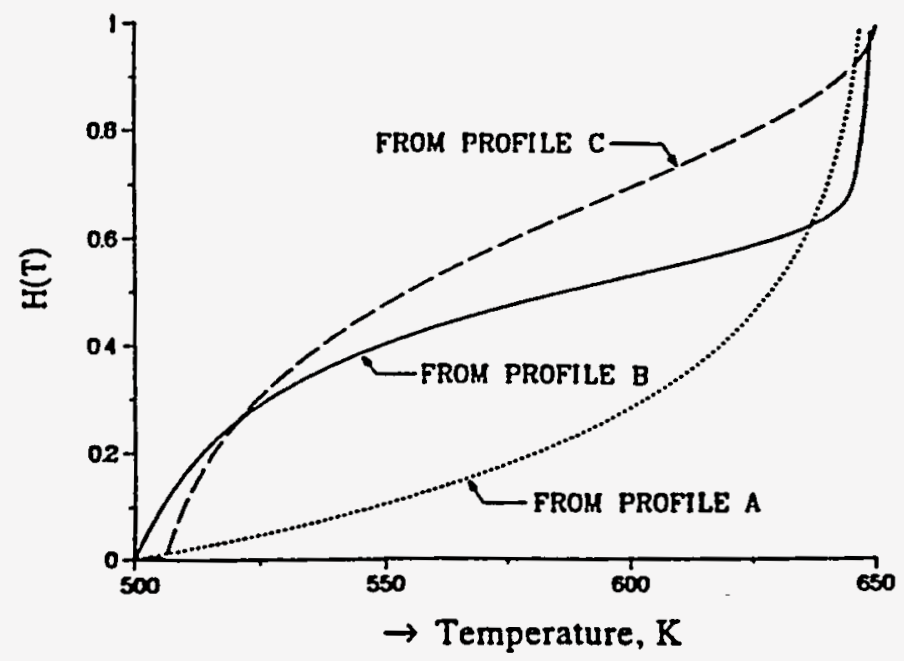

Figure 4. Cumulative temperature distributions corresponding to profiles in Fig. 2. A. Adiabatic rcactor with exothermic reaction or Hot Dry Rock reservoir with conduction-limited heat transfer. B. Highly fractured Hot Dry Rock reservoir. C. Plug-flow chemical reactor with exothermic reaction and external heat exchange.

\section{B. Inverting the Fredholm Equations}

1. The Three Fredholm Equations for Obtaining Temperature Profiles. In Sec. V, three FIEFKs appear whose solutions could give temperature profiles in flowing systems. These equations are

$$
\begin{aligned}
& g\left(E_{i}\right)=\int_{T_{\operatorname{man}}}^{T_{\max }} e^{-E_{j} / R T} \eta(T) d T \quad i=1,2, \ldots, n, \\
& e^{-E_{1} / R T_{\max }}-g\left(E_{1}\right)=\int_{T_{\min }}^{T_{\max }} \frac{E_{i}}{R T^{2}} e^{-E_{i} / R T} H(T) d T \quad i=1,2, \ldots, n,
\end{aligned}
$$

and

$$
g\left(E_{1}\right)-\int_{0}^{1} \exp \left(\frac{E_{i}}{R L_{0}(\xi)}\right) d \xi=\int_{0}^{1}\left(\frac{E_{i}}{R L_{0}(\xi)}\right)\left[\exp \left(\frac{E_{i}}{R L_{0}(\xi)}\right)\right] \varepsilon(\xi) d \xi \quad i=1,2, \ldots, n
$$


While these equations were derived for monotonic systems, Sec. VI shows that these equations are also valid for systems containing temperature extrema. Because these three equations are all FIEFKs, use of them will be considered together.

2. Approach to Testing the Different Factors. Each test first selected the temperature profile, then evaluated the RHS of Eq. (V-3), (V-7), or (V-11) for $n$ different activation energies within a given range. The synthetic data, $g\left(E_{i}\right)$, were then obtained by adding a specified amount of statistical error to each functional evaluation. All simulations used a grid of 20 points (i.e., $m=20$ ) to convert the system equation to discrete form. After solving the appropriate Fredholm integral equation to obtain the temperature profile, the calculated profile was compared with that originally postulated. All calculations used $q_{0}=q_{1}=1$ except those that compared using different values of $q_{0}$ and $q_{1}$.

3. Effect of Activation Energies. As stated earlier, the reaction activation energy $E_{i}$ is the only experimental variable for multiple-reaction tracer tests. Thus, an important consideration is the determination of the range of activation energies that best suits the chemical tracer experiments.

The required range of activation energies depends mainly on two factors, the kernel and the possible temperature profiles. In this case, the RHS of Eq. (V-11) decreases exponentially with $E_{i}$ for all three temperature profiles as $E_{i}$ changes from $20 \mathrm{~kJ} / \mathrm{mol}$ to $200 \mathrm{~kJ} / \mathrm{mol}$. It follows that the sensitivity of the tracer data to the activation energy (partial derivative of the RHS w.r.t. $E_{\mathfrak{j}}$ ) also decreases exponentially with the activation energy. Thus it is prudent to use tracers whose reactions have lower activation energies. Figure 5 depicts the results obtained by Method 3 with a grid of 20 points from $n=0$ to $n=1$, using five

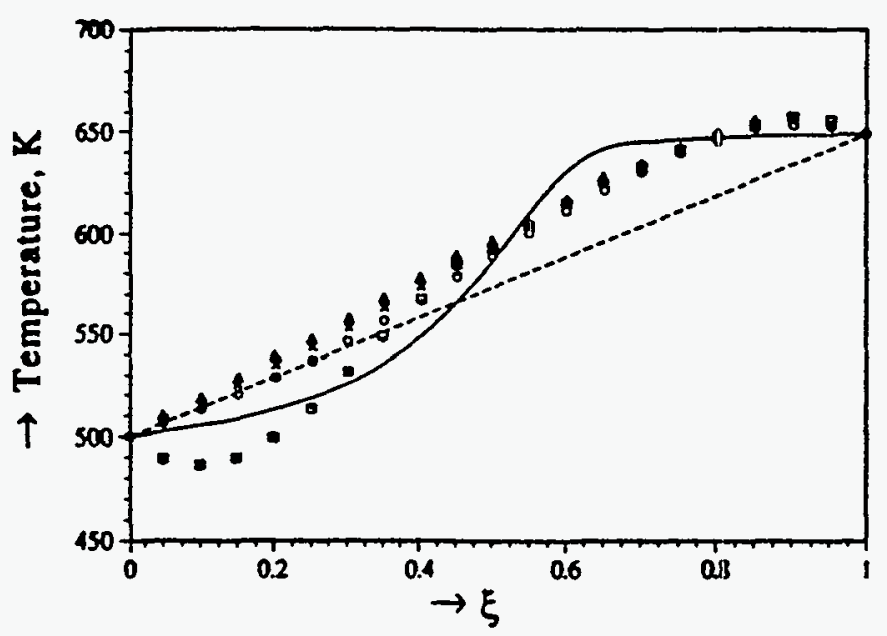

Figure 5: Effect of range of activation energy when temperature follows Profile B. Error $=1 \%$, tolerance $=0.01, q_{0}=q_{1}=1$, and $\alpha=1$. Solid curve shows the actual temperature profile and dashed curve depicts the initial estimate. Squares, triangles, $X ' s,+' s$, and circles represent the results obtained for activation-energy' ranges of 20-80, 80-140, 140-200, 40-200, and 40-46 $\mathrm{kJ} / \mathrm{mol}$, respectively. 
different ranges of activation energies, each consisting of seven data points (i.e., $n=7$ ). The solid curve is the true temperature profile (Profile B), the dashed line denotes the initial estimate of temperature profile (a straight line between known inlet and exit temperatures). The two ranges covering lower activation energies (viz., $20-80 \mathrm{~kJ} / \mathrm{mol}$ and $40-100 \mathrm{~kJ} / \mathrm{mol}$ ) gave better results than the two ranges of higher activation energies (viz., $80-140 \mathrm{~kJ} / \mathrm{mol}$ and 140-200 kJ/mol). Even when using lower activation energies, though, if the energies covered only a narrow range (e.g., $40-46 \mathrm{~kJ} / \mathrm{mol}$ ), the results suffered.

4. Effect of Number of Reacting Tracers. Once the range of activation energies is selected, an increase in the number of tracers beyond two within that range does not appear to be very important. Simulations shown in Fig. 6 illustrate this. The figure presents results obtained from matching Profile A when varying the number of data points from 2 to 13 . This test used $20-80 \mathrm{~kJ} / \mathrm{mol}$ for the range of activation energies. The figure clearly shows that the number of reactive tracers used does not markedly affect the results if the test uses at least two tracers. Still, when only 2 reactive tracers were used, the number of numerical iterations required increased significantly. Investigations of the effects of other variables used 4 or 5 data points to save computational efforts.

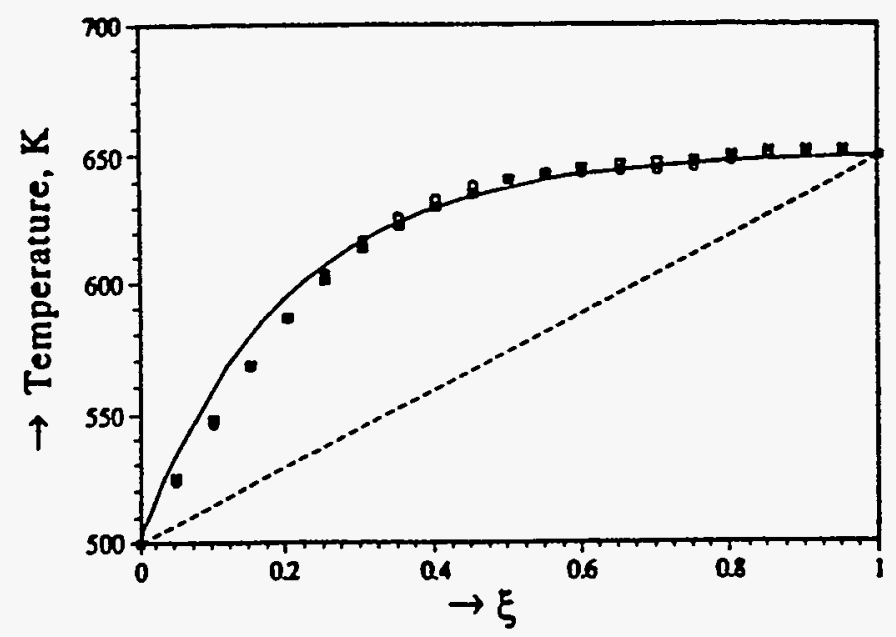

Figure 6: Effect of number of data points on the results. Error $=1 \%$, tolerance $=0.01, q_{0}=q_{1}=1$, and $\alpha=1$. Solid curve shows the actual temperature profile and dashed curve depicts the initial estimate. Squares, triangles, $\mathrm{XXX},+++$, and circles represent the results obtained for $\mathrm{n}=2,3,5,7$, and 13 , respectively.

5. Effect of Error Level in the Experimental Data. The studies discussed in the previous two paragraphs imposed no errors on the data. In any experiment, howvever, some error will inevitably occur, and the inversion of a FIEFK is an ill-posed problem. Thus a small level of error in the equation's LHS can cause large changes in the result if the solution technique takes no precautions. The success of the regularization methods used in this study depends on how robust are the methods with respect to the error 
level in the data values $\left[\left(q / A_{i} V_{s}\right) \ln \left(C_{i d} / C_{i 0}\right)\right.$ or $\left.g\left(E_{i}\right)\right]$. Figure $7 a$ shows that Method 3 passes this test. Three rms error levels $(0 \%, 1 \%$, and $5 \%)$ gave about the same results. Figure $7 \mathrm{~b}$ shows how well the method succeeded in distinguishing between Profiles A and B. Even with 10\% rms error levels in the data, the method readily distinguished between the two profiles. These profiles are the two competing models for a Hot Dry Rock geothermal reservoir. The calculations for recovering both temperature profiles assumed the same initial profile.
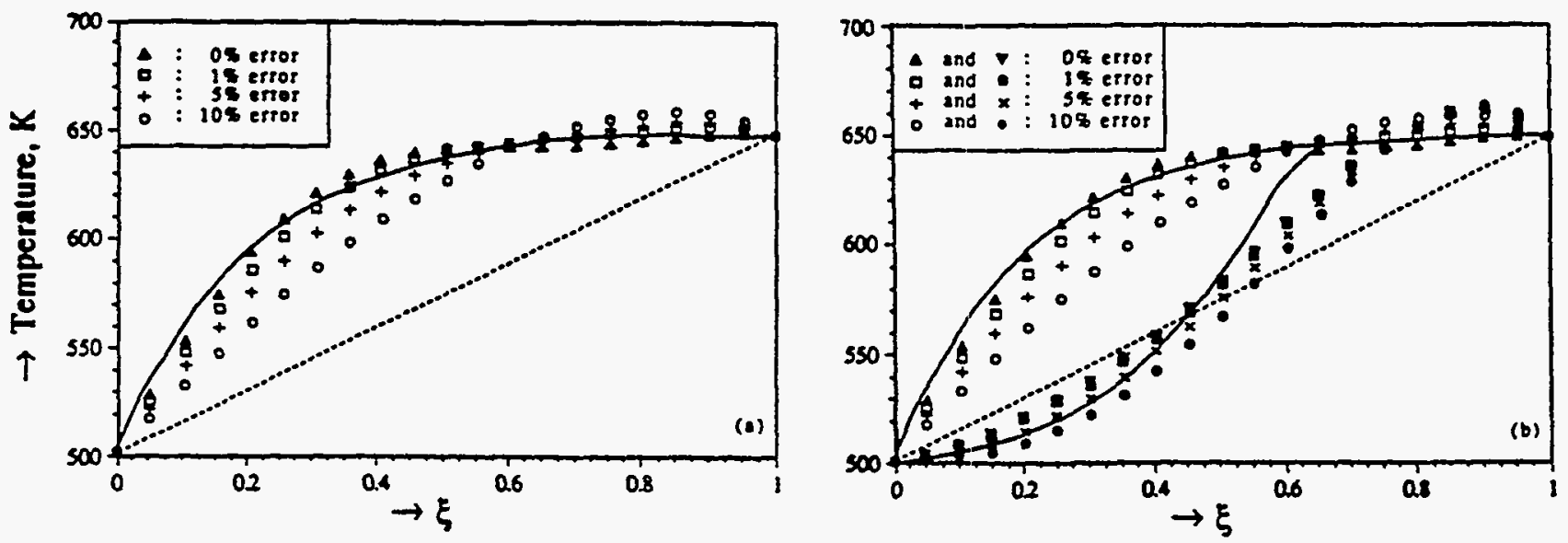

Figure 7: (a) Effect of error when temperature follows Profile A. (b) Discrimination between two rival profiles under various levels of error. $E_{\min }=40 \mathrm{~kJ} / \mathrm{mol}, E_{\max }=80 \mathrm{~kJ} / \mathrm{mol}, \mathrm{n}=5, q_{0}=q_{1}=1$, and $\alpha=1$.

Method 3 computes a modified temperature profile. Using this profile, the method then computes the RHS of Eq. (III-14) and compares it with the given data values $g\left(E_{i}\right)$. The method stops the iterations when both the norm of the new modifications in the temperature profile and the difference between the computer data and the given data fall below a specified tolerance level. The tolerance level is partially at the discretion of the user. The lower the tolerance, to a point, the better will usually be the results. Yet the tolerance should not drastically undershoot the error level in the data. Figure 8 underscores this point; the data there contain $1 \%$ rms error and it shows results for three levels of tolerance: $1 \%, 0.5 \%$, and $0.1 \%$ of the given data. The results improve when the tolerance level is decreased from $1 \%$ to $0.5 \%$. The results for the $0.1 \%$ tolerance level, however, show more distortion than when the two higher tolerance levels are used.

6. Comparing the Use of the Three Equations. In order to compare factors such as activation energy and error level, we need to use a single method. The results discussed so far have been obtained using only the third method with the first-order stabilizer and $q_{1}=q_{0}=1$. There are many forms of regularization that can be used in each method. For example, general regularization with a stabilizer of any 


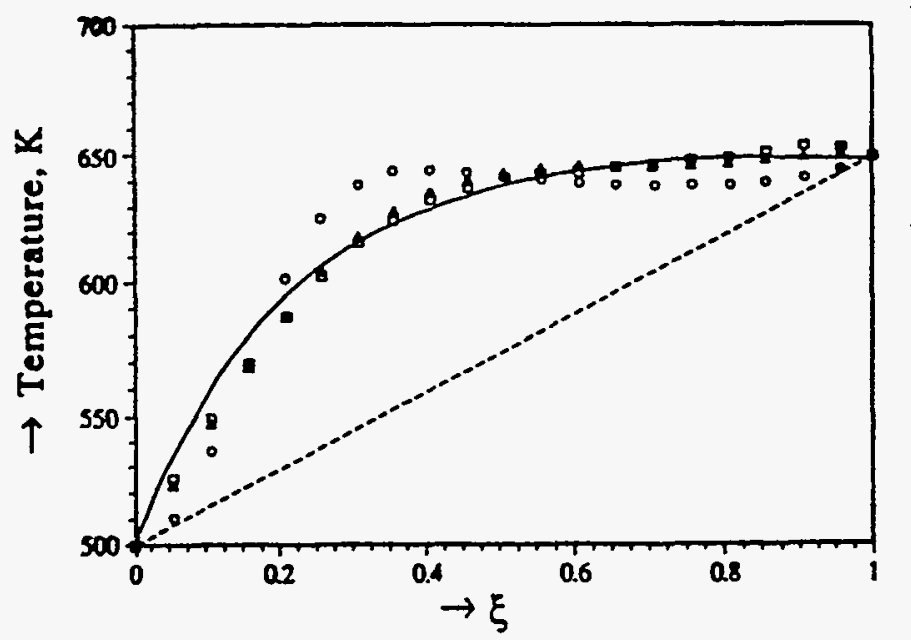

Figure 8: Effect of tolerance level on the results. $E_{\min }=20 \mathrm{~kJ} / \mathrm{mol}, E_{\max }=80 \mathrm{~kJ} / \mathrm{mol}, \mathfrak{n}=7$, error $=1 \%$, $q_{0}=q_{1}=1$, and $\alpha=1$. Solid curve shows the actual temperature profile and dashed curve depicts the initial estimate. Squares, triangles, and circles represent the results obtained for tolerances $=1 \%, 0.5 \%$, and $0.1 \%$ respectively. The number of iterations required in the three cases were 4,4 , and 10 , respectively.

order can be used. Using a first-order stabilizer furnishes an adjustable parameter $q_{1}$ additional to the one available when using a zero-order stabilizer. Depending on the temperature profile of interest, this additional parameter can be used to improve the results. Use of a first-order stabilizer results in smoothing of the first derivative of the unknown distribution $f$, besides a direct smoothing of $f$. Derivative smoothing does not constrain the form of $f$ as much as zero-order smoothing. Higher order stabilizers also can be used, though in this study we have not used second- or higher-order stabilizers.

Britten et al. (1983) reported an improvement in their results due to the non-negativity criterion in the BRD algorithm. For Methods 1 and 2 in this study; the results obtained with the BRD approach (using the zero-order stabilizer) were better than those obtained using general regularization. The latter method did not incorporate the non-negativity constraint, but could incorporate the boundary conditions. As mentioned earlier, the BRD method could not incorporate the boundary conditions. Figure 9 shows the strong influence of the non-negativity constraint on the results obtained by Method 2. The solid curve is the true Profile A. The BRD algorithm obtained the dotted curve. The dashed and chain-dot curves show the temperature profiles resulting from general regularization in Method 2 (with $q_{1}=q_{0}=1$ ). In obtaining the dashed curve, the boundary conditions were imposed; in obtaining the chain-dot curve, they were not imposed. Absence of the non-negativity condition did not affect the results drastically when boundary conditions were not imposed. Yet with boundary conditions imposed, the results without the non-negativity constraint overflowed the actual range of $\xi$. Summarizing the results from Fig. 9, Method 2 gives the best results when employing the $\mathrm{BRD}$ algorithm with the non-negativity constraint. 


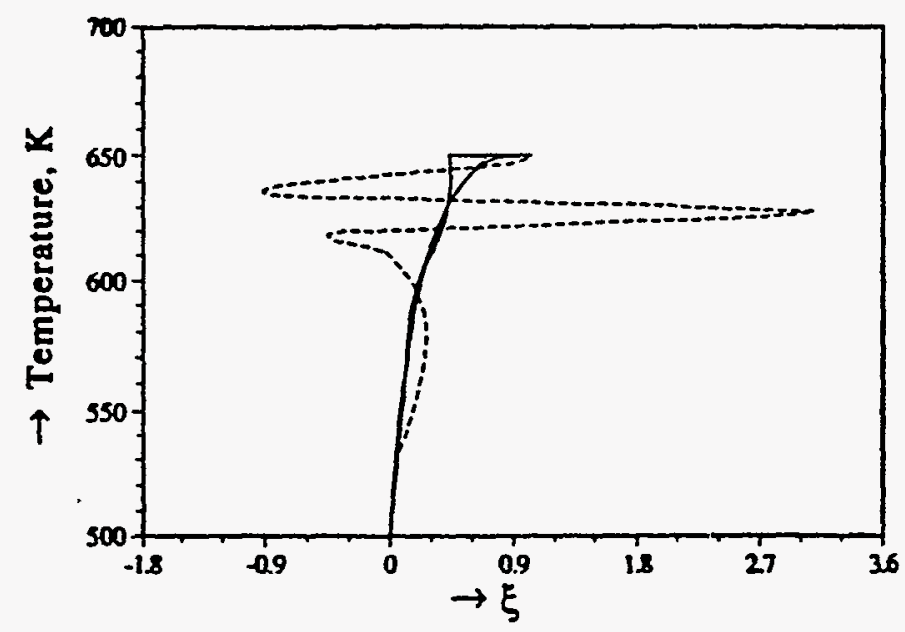

Figure 9: Effect of non-negativity constraint on the results from Method 2 for Profile A. Solid curve denotes the true profile whereas dotted, dashed, and chain dot curves show the results using the Butler algorithm, general regularization with boundary conditions, and general regularization without boundary conditions, respectively. $\left(q_{0}=q_{1}=1, n=7, E_{\min }=20 \mathrm{~kJ} / \mathrm{mol}, E_{\max }=80\right.$ $\mathrm{kJ} / \mathrm{mol}$, error $=0.1 \%$.)

Here the specified criterion to choose the "optimal" $\alpha$ did not yield the best results. Using general regularization constrained by the boundary conditions and $q_{0}=q_{1}=1$, the "optimal" $\alpha=10^{-7}$ obtained the dashed curve in Fig. 9. Better results were obtained using $\alpha=10^{-5}$. Yet results using the latter value of $\alpha$ also overflowed the range of $\xi$. This underscores the observacion that it is difficult to find a universal criterion for choosing the truly optimal $\alpha$ for regularization.

Method 3 can also use various forms of regularization. The BRD algorithm cannot use the nonnegativity constraint when incorporated into Method 3, because Method 3 inherently has the possibility of both positive and negative values in its distribution function. Figure 10 compares results from various forms of regularization when attempting to match Profile B using Method 3. The squares portray the results obtained when input data contained $1 \% \mathrm{rms}$ error, while circles represent the case of $5 \%$ error. Empty symbols denote first-order regularization $\left(q_{0}=q_{1}=1\right)$ and the solid symbols the BRD method that uses zero-order regularization. First-order regularization gave better results at both boundaries. Improvement is more prominent when the input data have a higher degree of error. Additional results from general regularization with a zero-order stabilizer (i.e., $q_{1}=0$, shown in the figure by symbol $X$ ) are inferior to the $B R D$ approach. The higher value of the ratio $q_{1} / q_{0}$ gave better results. Triangles in Fig. 10 show the results using $q_{0}=0, q_{1}=1$, and a $1 \% \mathrm{rms}$ error. There is a slight improvement from eliminating the smoothing of $f$ and having the solution depend entirely on the smoothing of $f$ s first derivative. Still, the improvement in the results is margirial when $q_{0}=0$ and $q_{1}=1$, and the number of 
iterations needed to reach the solution increases significantly. The simulations in this study therefore used $q_{0}=q_{1}=1$.

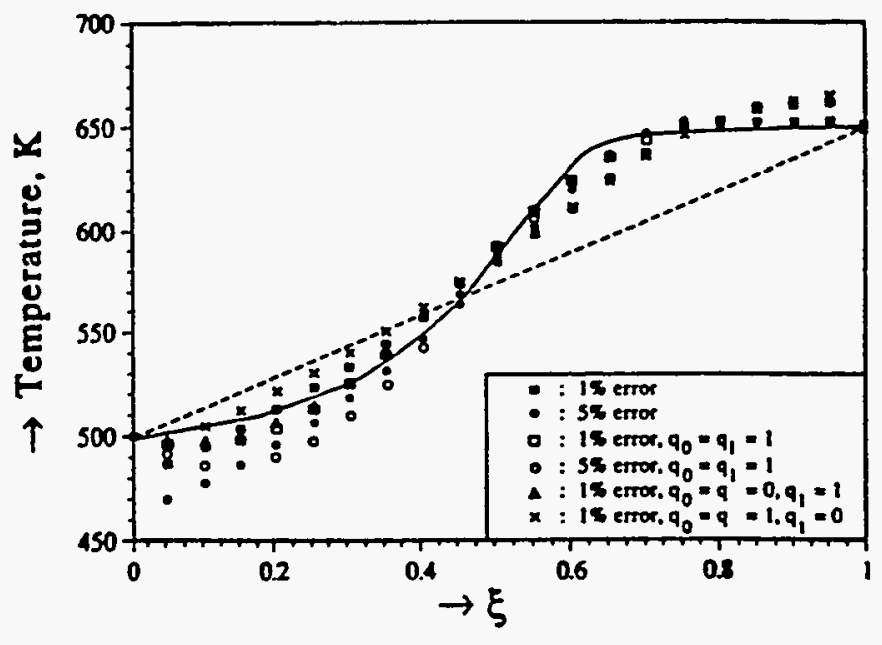

Figure 10: Comparison between the first-order regularization (empty symbols) and the Butler algorithm (solid symbols). ( $q_{0}=q_{1}=1, n=7, E_{\min }=20 \mathrm{~kJ} / \mathrm{mol}, E_{\max }=80 \mathrm{~kJ} / \mathrm{mol}$, error $=0.1 \%$.)

The results from Figs. 9 and 10 make a comparison among the three methods difficult. Methods 1 and 2 furnish the best results with the BRD approach that uses the non-negativity criterion. Yet Method 3 cannot incorporate the non-negativity criterion. Since Method 3 has used general regularization in obtaining most of the results presented earlier, we could compare results from Method 3 using general regularization with results from Methods 1 and 2 using general regularization. Unfortunately; as Fig. 9 illustrates, general regularization does not give the best results for Method 2. (Method 1 elicited a similar observation.) Instead of comparing the three methods using general regularization, knowing that general regularization is not the best approach for Methods $I$ and 2, we decided to compare the best results we obtained from each method. Therefore, for our comparison, Methods 1 and 2 use the BRD approach with the non-negativity constraint and Method 3 uses general regularization with $q_{0}=q_{1}=1$.

Figure 11 compares the three methods for the temperature profiles A and B. Again the solid curve represents the actual temperature profile. Dashed and dotted curves show the results obtained by Methods 1 and 2, respectively. These curves are drawn smoothly through the solution obtained at a discrete number of grid points. As stated earlier, these methods used the BRD approach with the non-negativity criterion and the zero-order stabilizer. For Method 3, since the non-negativity criterion does not apply, results were obtained by two approaches. The indirect BRD method without their non-negativity criterion obtained the results denoted by circles. Triangles are the results from the traditional regularization method with a firstorder stabilizer. All simulations presented in Fig. 11 added 3\% rms errors to the input data points, and 1\% 
tolerance was used to stop the iterative procedure. The calculations reported in Figs. 1la and 11c used five equally spaced data points corresponding to the activation energies over the $40-80 \mathrm{~kJ} / \mathrm{mol}$ range spaced at $10 \mathrm{~kJ} / \mathrm{mol}$ intervals. For Profile A (Fig. 11a), all three methods give acceptable results. For Profile B with its inflection point, however, the third method with general regularization yields the best results. Figures $1 \mathrm{lb}$ and $1 \mathrm{ld}$ show the comparison in a more severe case, in which only two data points (activation energies of 40 and $80 \mathrm{~kJ} / \mathrm{mol}$ ) are used for the computations. Here, Method 1 is markedly inferior with respect to other methods. Methods 1 and 2 solve FIEFKs to obtain $\eta(T)$ and $H(T)$, and they then determine $T(\xi)$. This indirect approach can lead to a temperature profile that does not start at $\xi=0$ (Method 2), or to a profile that does not end at $\xi=1$ (Methods 1,2).
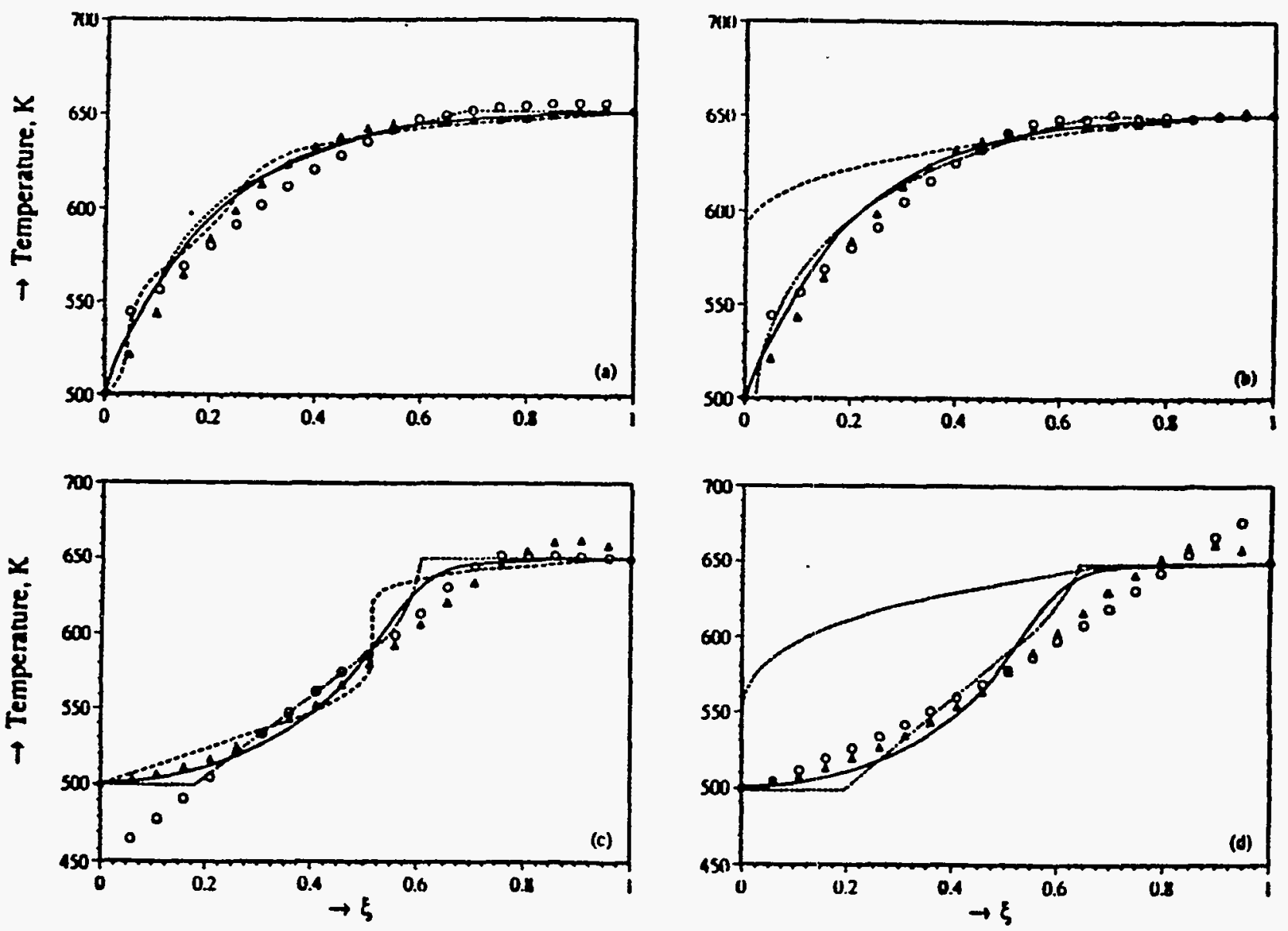

Figure 11: Comparison of various methods. Solid curves depict the actual temperature profile. Dashed and dotted curves show the results obtained by Methods 1 and 2, respectively. Triangles represent the results of traditional regularization approach (Method 3), whereas circles represent the results of Butler approach (Method 3) without the non-negativity criterion. $E_{\min }=40 \mathrm{~kJ} / \mathrm{mol}, E_{\max }=80 \mathrm{~kJ} / \mathrm{mol}$, error $=3 \%$; for Method 3, tolerance $=0.01, q_{0}=q_{1}=1$, and $\alpha=1$. 
For smooth and monotonic temperature profiles, Method 1 does not give solutions as close to the true profiles as do Methods 2 and 3. When there are few data points, errors in Method 1 solutions can be extremely serious. Methods 2 and 3, on the other hand, appear acceptably robust.

In normal regularization the choice of the regularization smoothing parameter is important. Yet in this new iterative scheme, the regularization parameter selected for each iteration does not affect the results to a significant degree. Selection of a very large $\alpha$ reduces the possibility of introducing spurious structure, though it slows the approach to the solution. Decreasing the value of $\alpha$ accelerates convergence to the solution until some critical value is reached. Figure 12 illustrates this point for Profile A. For a very low value of $\alpha$ (here, $10^{-7}$ ), the regularization loses its smoothing capacity and therefore the solution contains undesired spurious structures. For still lower values of $\alpha$, desired tolerance is never achieved.

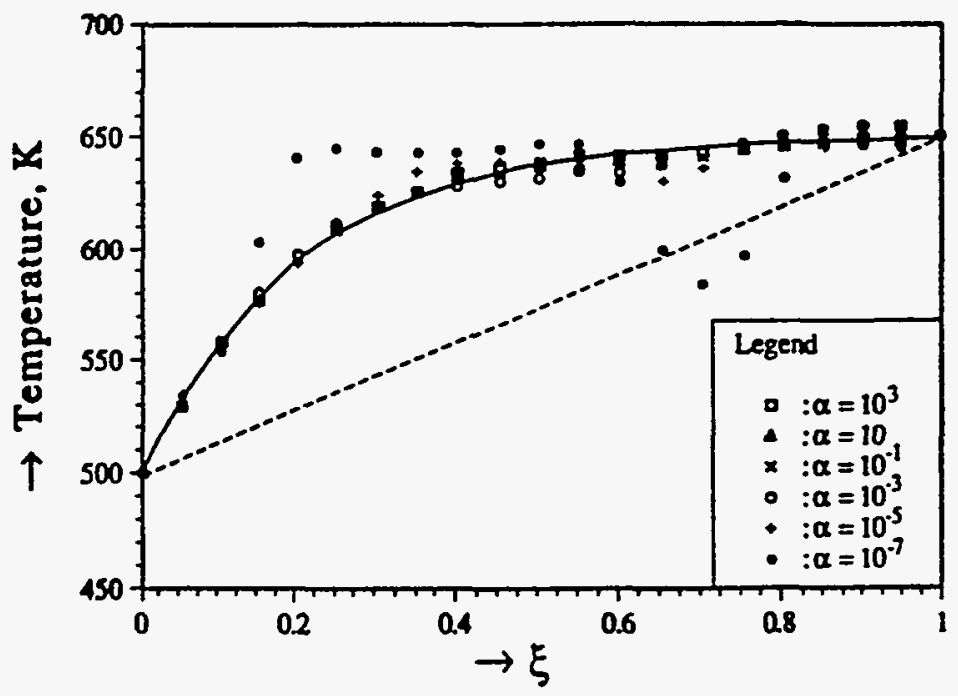

Figure 12: Effect of the regularization parameter $\alpha$ on the results from Method 3. $\left(q_{0}=q_{1}=1, n=7\right.$, $E_{\min }=20 \mathrm{~kJ} / \mathrm{mol}, E_{\max }=80 \mathrm{~kJ} / \mathrm{mol}$, error $=0.1 \%$.)

7. Temperature Profiles Exhibiting a Maximum. To test Method 3 for cases with a peak in the temperature profile, Profile $C$ was used for three sets of $\beta_{1}$ and $\beta_{2}$ values. (Table 1 gives the three sets of values.) Figure 13 shows the results. For Profiles $\mathrm{Cl}$ and $\mathrm{C} 2$, Method 3 gives good results, which Fig. 13 depicts by empty squares and circles, respectively. In the third case, the results from the regularization (shown by the solid squares) do not agree with the actual Profile $\mathrm{C} 3$, although the tolerance of $0.5 \%$ is satisfied. The computed data values in Table 2 show that, in this situation at least, several possible temperature profiles can be obtained from the same experimental data. Thus, in the cases where multiple solutions are possible, the solution obtained by regularization depends on the temperature profile assumed for the first iteration. Figure 14 shows that many significantly different solutions, all valid, can be 
obtained by varying the initial estimate. In that figure, the thick solid curve represents the true profile, whereas the other five curves are five solutions starting from different initial estimates. The underlying reason behind these multiple solutions is that regularization finds the smoothest solution. For monotonic profiles, no solution but the true solution will be as smooth. In other cases, if the true profile has a maximum and the boundary conditions are equal, there can be other solutions that are as smooth as the actual profile. Regularization is unable to distinguish one from another in this situation.

\section{Table 1}

CHARACTERISTICS OF PROFILES WITH EXTFEMA USED FOR TESTING

$\begin{array}{cccccc}\begin{array}{c}\text { Profile } \\ \text { number }\end{array} & \beta_{1} & \beta_{2} & \mathrm{~T}_{\max } & \begin{array}{c}\xi \text { at which } \\ \mathrm{T}=\mathrm{T}_{\max }\end{array} & \mathrm{T} \text { at } \xi=1 \\ \mathrm{C} 1 & 3.398 & 1.25 & 650 & 0.8000 & 646.0 \\ \mathrm{C} 2 & 5.437 & 2.00 & 650 & 0.5000 & 610.4 \\ \mathrm{C} 3 & 16.31 & 6.00 & 650 & 0.1667 & 506.1\end{array}$

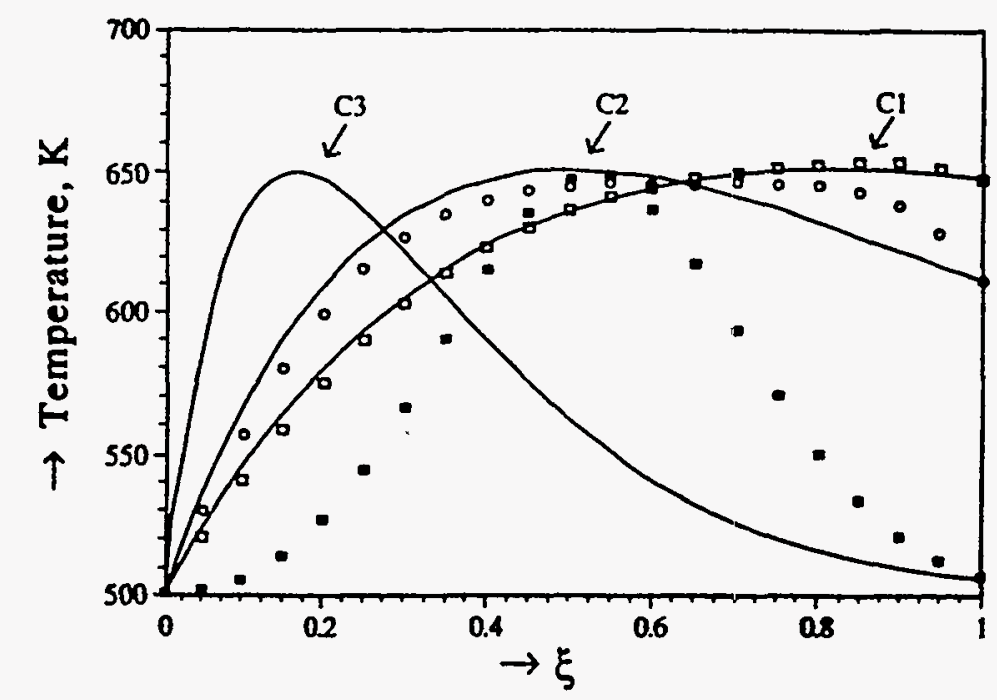

Figure 13: Inversion of temperature profiles exhibiting peaks. Empty squares, circles, and solid squares represent results for Profiles $\mathrm{Cl}, \mathrm{C2}$, and $\mathrm{C} 3$, respectively. $\mathrm{E}_{\mathrm{mn}}=40 \mathrm{~kJ} / \mathrm{mol}$, $E_{\max }=80 \mathrm{~kJ} / \mathrm{mol}$, error $=1 \%$, and $q_{0}=q_{1}=1$, and $\alpha=1$. 
Table 2

\section{COMPARISON OF DATA AND RHS OF EQ. (III-14) IN ATTEMPT TO RECOVER PROFILE C3 PICTURED IN FIG. 13}

\begin{abstract}
Activation energy, $E_{1}$ $(\mathrm{kJ} / \mathrm{mol})$
\end{abstract}

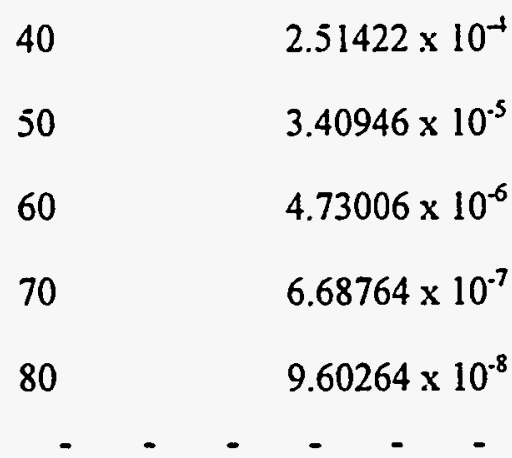

$$
g\left(E_{i}\right) \text {, }
$$$$
\text { with error }
$$

$2.43014 \times 10^{-4}$

$3.42493 \times 10^{-5}$

$4.68331 \times 10^{-6}$

$6.77676 \times 10^{-7}$

$9.78366 \times 10^{-8}$
Data simulated from computed temperature profile

$2.4961 \times 10^{-4}$

$3.38032 \times 10^{-5}$

$4.68022 \times 10^{-6}$

$6.59878 \times 10^{-7}$

$9.44147 \times 10^{-8}$

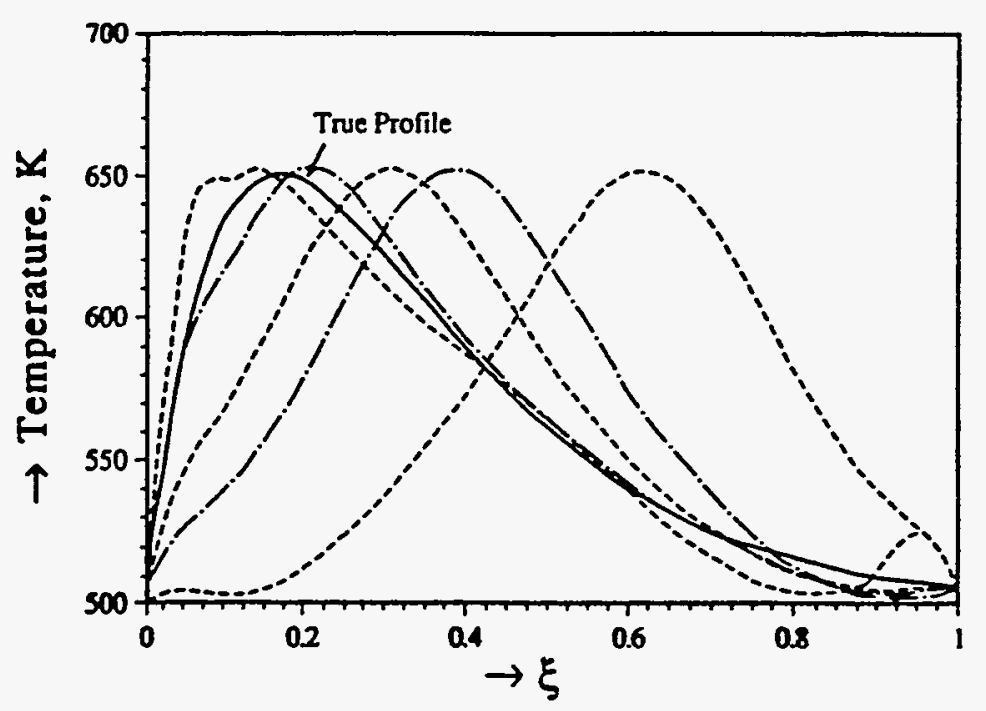

Figure 14: Results for Temperature Profile C3, obtained using various initial estimates.

If $T(\xi)$ is the actual temperature profile, then other profiles, such as the following $U(\xi)$,

$$
\mathrm{U}(\xi)=\mathrm{T}\left(\xi-\xi_{0}\right) \text { if } \xi_{0} \leq \xi \leq 1 \text {, and } \mathrm{U}(\xi)=\mathrm{T}\left(1+\xi-\xi_{0}\right) \text { if } 0 \leq \xi \leq \xi_{0} \text {. }
$$

can be constructed that will cause the same experimental observations [i.e., the LHS of Eq. (III-14)]. A temperature profile can be cut into many segments, and every temperature profile obtained by reassembling these segments in any sequence will furnish the same values for the LHS of Eq. (III-14). Yet many of these profiles would not be valid solutions to the problem because they would not satisfy the boundary 
conditions or the solutions would contain high-frequency components. Even if the boundary conditions at both ends are equal, then for every temperature profile satisfying the FIEFK, its mirror image also will be a solution. Both are equally smooth.

Apparently, widely differing multiple solutions are possible if the true temperature profile exhibits a sharp peak and the temperatures at the split boundaries are not far apart. Under these conditions, it is possible to reassemble the segments of the profile and still observe the boundary conditions and continuity of the profile. For monotonic profiles such as Profiles $\mathrm{A}$ and $\mathrm{B}$, multiple solutions do not occur, irrespective of the initial estimate. Figure 15 illustrates two such cases. Poor initial estimates, shown in the figure by curves IC-A and IC-B, are used for the two profiles. Even so, Method 3 distinguishes between the two profiles. The squares depict the results obtained for Profile A from the initial estimate IC-A. The triangles represent the analogous results for Profile B. Though the results are inferior to those obtained using a good initial estimate, it is still possible to distinguish between the two profiles.

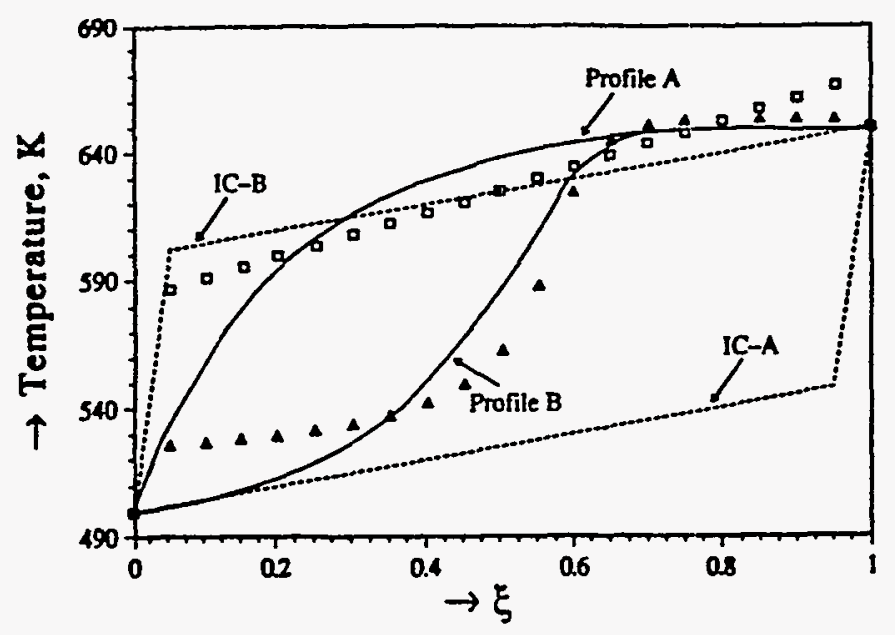

Figure 15: Performance of Method 3 with poor initial estimates in the cases of Temperature Profiles A and $B$. Squares and triangles represent rcsults obtained from initial profiles IC-A and IC-B, respectively. $\mathrm{n}=7$, tolerance $=0.005, \mathrm{E}_{\min }=20 \mathrm{~kJ} / \mathrm{mol}, \mathrm{E}_{\max }=80 \mathrm{~kJ} / \mathrm{mol}, \mathrm{q}_{0}=1, q_{1}=0$, $\alpha=100$.

\section{DISCUSSION}

Earlier publications have discussed experimental aspects of this technique (Robinson et al. 1984, 1988). The data consist of entering and exit concentrations of the reacting tracers, and these combine to form the vector of experimental data $g\left(E_{i}\right)$. Either Method 2 or Method 3 uses this vector to obtain a temperature profile for the system.

Method 2 requires a criterion for the optimal value of the smoothing parameter $\alpha$, while Method 3 does not. Method 3, on the other hand, requires an initial guess for the profile. A comparison of Fig. 15 
with the crosses in Fig. 6 shows that a very poor initial guess can give a somewhat poorer result. Still, Method 3 is probably to be preferred because it does not require the optimal value of the smoothing parameter.

It is necessary to judge the precision and accuracy of a calculated temperature profile. In the numerical experiments reported here, of course, the true profile was known; in a practical application, the goal is to obtain the unknown profile. To judge the precision and accuracy with the present state of the art, numerical experiments of the type reported here are necessary using the calculated profile. Equation (III-14) would be solved to obtain synthetic data using the calculated profile and using profiles that deviate stipulated amounts from the calculated profile. Method 2 or 3 would then invert Eq. (III-14) to obtain profiles corresponding to the sets of synthetic data. This procedure would measure the precision obtainable for the situation being evaluated. To judge the accuracy, numerical experiments would be carried out for different levels of error in the synthetic data. The accuracy in the profile obtained for the error level existing in the actual data would be the accuracy of the result.

When a calculated temperature profile possesses temperatures significantly higher or lower than both inlet and outlet temperatures, an extremum is present and multiple solutions are possible, as discussed earlier. Figure 14 shows that when multiple solutions exist, the calculated profile depends significantly on the initial guess. For an accurate temperature profile in this case, further information will most likely be necessary. If the location of the extremum can be specified, this is probably sufficient. Whereas it may be more difficult to obtain an accurate profile when an extremum is present, the mere discovery of the extremum's existence may justify the use of the method.

\section{PRACTICAL IMPLEMENTATION OF THE PRINCIPLES}

\section{A. Characteristics of Suitable Tracers}

Among the requirements for suitable tracer reactants and products are those that are common to all tracers. They must be soluble in the system medium, neither reactants nor products can adsorb appreciably on the system surfaces, and capability must be present for easy, quick, and accurate analysis of the effluent for reactant and product concentrations.

Besides these general tracer requirements, the reaction or reactions must possess appropriate preexponential factors and activation energies. The rate expressions characterizing the reactions must also be sufficiently tractable that they lend themselves to analysis of the results. The amount of heat generated or absorbed by the tracer reaction or reactions must be sufficiently small that the temperature field of the system is not affected significantly. This means either that the tracer reactions' heats of reaction must be small enough to meet this requirement or that the reactants' concentrations are low enough to meet the requirement. 
For multiple-reaction tracer tests, one must use a suite of reactions with a spread of activation energies. The pre-exponential factors must be such that the conversions for the reactions are all in the range where the sensitivity is acceptable. There must be sufficient reactions to achieve the desired precision in the temperature distribution.

\section{B. Temperatures and Temperature Measurement in Hot Dry Rock Geothermal Reservoirs}

To date, attempts to use the reactive tracer idea have been limited to HDR geothermal reservoirs. Thus the reactions chosen have kinetics appropriate for this application. Two classes of reactions have been proposed: the hydrolysis of organic esters and amides (Robinson et al. 1988), and the hydrolysis of derivatives of bromobenzene (Birdsell and Robinson 1989). It is desirable to select classes of reactions with similar mechanisms within the class. Then altering the nature of the substrate in a systematic way can produce reactions with a range of values of the kinetics parameters. A complication of both these reaction classes is that the reaction rates are strong functions of the hydroxide ion concentration in natural geofluids. The hydroxide ion concentration in turn is a strong function of temperature, but it does not change much as the tracer reaction progresses. For the conditions proposed for HDR reservoir experiments, these phenomena create a pseudo-first-order reaction. In these reactions, the kinetics are functions of the intrinsic second order rate constant and the temperature-dependent hydroxide ion concentration. This must be modeled based on the dissolved ion content of the fluid and the measured room-temperature $\mathrm{pH}$. An analysis by Robinson and Birdsell (1987) showed that for typical geofluids and tracer transit times, the ester and amide hydrolysis reactions are appropriate as reactive tracers in the temperature range $75-100^{\circ} \mathrm{C}$, while the bromobenzene reactions are valid in the temperature range of approximately $150-275^{\circ} \mathrm{C}$.

\section{CONCLUSIONS}

1. It is possible to measure temperature profiles in plug-flow systems using chemically reacting tracers.

2. It is possible to measure temperature histories in perfectly mixed batch systems using chemically reacting tracers.

The remaining conclusions, though referring to temperature profiles and plug-flow systems, can also be applied to temperature histories and perfectly mixed batch systems.

3. When only a single parameter in the temperature distribution is needed, a single-tracer test may furnish this information.

4. Conversion of reacting tracers in a plug-flow system involves an integral equation. Analysis of multiple-tracer tests requires solution of the integral equation. Three different methods can convert this integral equation into a FIEFK. In the first method, the function to be determined is a temperature distribution function. The second method employs a cumulative temperature 
distribution function in the development of the FIEFK. The third method, an iterative one, uses a Taylor series expansion of the kernel exponential function in terms of a correction to a postulated temperature profile.

5. An alternative to conversion of the integral equation into a FIEFK is the use of an assumed temperature-distribution function containing adjustable parameters. Data from the tracer tests are used to optimize the parameters in the distribution.

6. The technique of regularization can be used to solve the FIEFKs developed in this work. In the first two methods, a criterion must be selected for determining the optimal value of the smoothing parameter, in common with most applications of regularization for solving FIEFKs. For this work the optimal smoothing parameter usually followed the criterion that the optimal parameter gave the lowest sum of squared differences between the experimental and calculated points. Literature studies suggest that this criterion is not generally useful, so this conclusion probably applies only to the type of situations studied here.

7. In the third method, the iterative one, the choice of the regularization smoothing parameter is not critical if it is sufficiently large to damp out spurious oscillations.

8. The sole experimental variable in the equations developed for determining temperature profiles is the tracer-reaction activation energy. Thus, tracer reactions with different activation energies must be used to gain information.

9. If temperature extrema are present within the system, multiple solutions to the FIEFK may be possible. Even when multiple solutions are possible, valuable information such as the existence of system hot spots may be obtained.

10. The results are more sensitive to a change in reaction activation energy when the activation energy is low than when it is high. For a given range in activation energies, it is better to use tracer reactions with lower activation energies.

11. The temperature profiles obtained using the methods developed here are not particularly sensitive to error in the data. Little difference was detected in the profiles obtained from data containing $0-5 \% \mathrm{rms}$ error in the data. Good profiles could be obtained from data containing as much as $10 \% \mathrm{rms}$ error in the data.

12. In a comparison of the quality of the three methods for obtaining temperature profiles, Methods 2 and 3 gave solutions of comparable quality. Method 1 on occasion gave results significantly inferior to those obtained using Methods 2 and 3. Method 2 still possesses the difficulty of determining the optimal value of the regularization smoothing parameter.

13. For two types of monotonic temperature profiles examined in this work, an adequate temperature profile may be extracted using as few as two reacting tracers. This can distinguish between the two models with as much as $10 \% \mathrm{rms}$ error in the data. 
14. Ester and amide hydrolysis reactions are valid HDR tracer reactions for measuring temperatures in the range $75-100^{\circ} \mathrm{C}$. Hydrolyses of bromobenzene derivatives are valid HDR tracer reactions for measuring temperatures in the range $150-275^{\circ} \mathrm{C}$.

\section{ACKNOWLEDGMENTS}

C. 0 . Grigsby contributed much in helpful discussions. This work was carried out under the auspices of the U. S. Department of Energy.

\section{REFERENCES}

Allison, H., "Inverse Unstable Problems and Some of Their Applications," Math. Scientist, 4, 9-30 (1979).

Arrhenius, S., "Über den Reaktiongeschwindigkeit bei der Inversion von Rohrzucker durch Säuren," $Z$. phys. Chem., 4, 226-248 (1889).

Benson, S. W., The Foundations of Chemical Kinetics, pp. 66-67. McGraw-Hill, New York, 1960.

Bodenstein, M. "Die Ermittlung des Mechanismus chemischer Reaktionen," Helv. Chim. Acta, 18, 743-759 (1935).

Birdsell, S. A., and B. A. Robinson, "Kinetics of Aryl Halide Hydrolysis Using Isothermal and Temperature-Programmed Reaction Analyses," Ind. Chem. Chem. Res., 28, 511 1-518 (1989).

Bischke, S. D., R. Chemburkar, L. F. Brown, and B. J. Travis, "The Extraction of Site-Energy Distributions from Temperature-Programmed Desorption Spectra," in Fundamentals of Adsorption (A. B. Mersmann and S. E. Scholl, eds.), pp. 145-154. Engineering Doundation, New York (1991).

Britten, J. A., B. J. Travis, and L. F. Brown, "Calculation of Surface Site-Energy Distributions from Adsorption Isotherms and Temperature-Programmed Desorption Data," AIChE Symp. Ser. No. 230, 79, 7-17 (1983).

Brown, L. F., and B. J. Travis, "Optimal Smoothing of Site-Energy Distributions from Adsorption Isotherms," in Fundamentals of Adsorption (A. L. Myers and G. Belfort, eds.), pp. 125-134. Engineering Foundation, New York, 1984.

Brown, L. F., B. A. Robinson, and J. W. Tester, "Determination of Temperature Characteristics of Flowing Systems Using Chemically Reacting Tracers," paper 67c presented at 1984 Summer AIChE Meeting, Philadelphia, PA. August 19-22, 1984.

Butler, J. P., J. A. Reeds, and S. V. Dawson, "Estimating Solutions of First Kind Integral Equations with Nonnegative Constraints and Optimal Smoothing," SIAMJ. Numer. Anal., 18, 381-397 (1981).

Chemburkar, R. M., L. F. Brown, B. J. Travis, and B. A. Robinson, "Numerical Determination of Temperature Profiles in Flowing Systems from Conversions of Chemically Reacting Tracers," Chem. Eng. Sci., 46, 1349-1360 (1991).

Dash, Z. V., H. D. Murphy, R. L. Aamodt, R. G. Aguilar, D. W. Brown, D. A. Counce, H. N. Fisher, C. O. Grigsby, H. Keppler, A. W. Laughlin, R. M. Potter, J. W. Tester, P. E. Trujillo, and G. A. Zyvoloski, "Hot Dry Rock Geothermal Reservoir Testing: 1978-1980," J. Volcan. Geoth. Res., 15, 7145-7158. (1983).

Edgar, R. G., and D. M. Himmelblau, Optimization of Chemical Processes. McGraw-Hill, New York, 1988. 
Fogler, H. S., and L. F. Brown, "Distributions of Residence Times for Chemical Reactors," Chap. 13 in Elements of Chemical Reaction Engineering (by H. S. Fogler), pp. 708-758. Prentice-Hall, Englewood Cliffs, NJ, 1992a.

Fogler, H. S., and L. F. Brown, "Analysis of Nonideal Reactors," Chap. 14 in Elements of Chemical Reaction Engineering (by H. S. Fogler), pp. 759-803. Prentice-Hall, Englewood Cliffs, NJ, 1992b.

Gill, P. E., W. Murray and M. H. Wright, Practical Optimization, p. 134 ff. Academic Press, New York, 1981.

Glasstone, S., Textbook of Physical Chemistry, pp. 1088-89. Van Nostrand, Princeton, NJ, 1946.

Koch, E., Non-Isothermal Reaction Analysis, pp. 4-5. Academic Press, New York, 1977.

Miller, G. F., "Fredholm Equations of the First Kind," in Numerical Solution of Integral Equations (L. M. Delves and J. Walsh, eds.), pp. 175-187. Clarendon Press, Oxford, 1974.

Murphy, H. D., J. W. Tester, C. O. Grigsby, and R. M. Potter, "Energy Extraction from Fractured Geothermal Reservoirs in Low Permeability Crystalline Granite," J. Geophysical Res., 86(B8), 7145-7158 (1981).

Nauman, E. B., and B. A. Buffham, Mixing in Chemical Reactors. Wiley, New York, 1983.

Nicol, D. A. C., and B. A. Robinson, "Modeling the Heat Extraction from the Rosemanowes HDR Reservoir," Geothermics, 19(3), 247-257 (1990).

Riggs, J. B., An Introduction to Numerical Methods for Chemical Engineers. Texas Tech. Univ. Press, Lubbock, TX, 1988.

Robinson, B. A., and S. A. Birdsell, "Tracking Thermal Fronts with Temperature-Sensitive, Chemically Reactive Tracers," presented at Geothermal Resources Council Annual Mecting, Sparks, NV October 11-14, 1987.

Robinson, B. A., and G. F. Jones, "A Tracer Based Model for Heat Extraction in a Hot Dry Rock Reservoir," Trans. Geothermal Resources Council. 11, 615-622 (1987).

Robinson, B. A., J. W. Tester, and L. F. Brown, "Using Chemically Reactive Tracers to Determine Temperature Characteristics of Geothermal Reservoirs," Trans. Geothermal Resources Council, 8, 337-341 (1984).

Robinson, B. A., J. W. Tester and L. F. Brown, "Reservoir Sizing Using Chemically Reactive Tracers," SPE Formation Evaluation, 227-234 (March, 1988).

Smith, J. M., Chemical Engineering Kinetics (2nd ed.), pp. 522-523. McGraw Hill, New York, 1970.

v. Szombathely, M., P. Bräuer, and M. Jaroniec, "The Solution of Adsorption Integral Equations by Means of the Regularization Method," J. Comput. Chem., 13, 17-32 (1992).

Tikhonov, A. N., and V. Y. Arsenin, Solutions of Ill-Posed Problems. Halsted Press, 1977.

Wen, C.-Y., and L. T. Fan ${ }_{\circ}$ Models for Flow Systems and Chemical Reactors, pp. 60-63. Dekker, New York, 1975.

Wing, M., "A Primer on Integral Equations of the First Kind," Report LA-UR-84-1234, Los Alamos National Laboratory, Los Alamos, NM (1984). 


\section{APPENDIX A}

\section{PARAMETER PRECISION IN LEAST-SQUARES OPTIMIZATION}

The conditions for reaching a minimum in a function $\phi\left(\beta_{1}, \beta_{2}, . . \beta_{j} . ., \beta_{m}\right)$ are

$$
\frac{\partial \phi}{\partial \beta_{j}}=0 \quad \mathrm{j}=1,2, \ldots, \mathrm{m} .
$$

Yet in numerical evaluation of the minimum, this ideal is not reached. The minimum is approximated when

$$
\left|\frac{\partial \phi}{\partial \beta_{j}}\right|<\varepsilon j=1,2, \ldots, m,
$$

in which $\varepsilon$ is some very small predetermined value. Assuming that $\partial^{2} \phi / \partial \beta_{i}{ }^{2}$ is approximately constant in the region between $\left|\partial \phi / \partial \beta_{j}\right|=\varepsilon$ and $\left(\partial \phi / \partial \beta_{j}\right)=0$, or assuming that

$$
\frac{\partial^{2} \phi}{\partial \beta_{j}^{2}} \approx \gamma \quad-\varepsilon<(\partial \phi / \partial \beta)<+\varepsilon,
$$

$\gamma$ being a constant. The change in $\partial \phi / \partial \beta_{j}$ between the point where $\left(\partial \phi / \partial \beta_{j}\right)=0$ and the points where $\left|\partial \phi / \partial \beta_{\mathrm{j}}\right|=\varepsilon$ is of course $\varepsilon$, but is also approximately equal to

$$
\delta\left(\frac{\partial \phi}{\partial \beta_{\mathrm{j}}}\right) \approx \frac{\partial^{2} \phi}{\partial \beta_{\mathrm{j}}^{2}} \delta \beta_{\mathrm{j}} \approx \gamma \delta \beta_{\mathrm{j}} .
$$

As a result,

$$
\gamma \delta \beta_{\mathrm{j}} \approx \varepsilon, \text { or } \delta \beta_{\mathrm{j}} \approx \varepsilon / \gamma
$$

This says that within the region between the minimum point where $\beta_{i}$ assumes its "best" value and the line where $\left|\partial \phi / \partial \beta_{i}\right|=\varepsilon$, the greatest change possible in the parameter $\beta_{i}$ is $\varepsilon / \gamma$. Since the value of the parameter $\beta_{i}$ can vary over this region and the minimization function is still judged to be at its minimum value, the precision obtainable in $\beta_{i}$ is equal to plus or minus the $\delta \beta_{i}$ calculated in Eq. (A-5). The higher the value of $\gamma$, i.e., the higher the value of the second derivative of Eq. (A-3), the lower the value of $\delta \beta_{j}$ and the greater the precision in $\beta_{\mathrm{j}}$. 


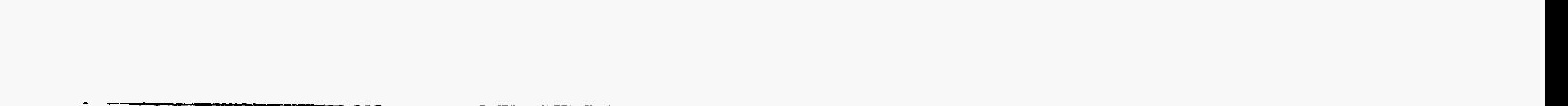




\section{APPENDIX B \\ DEVELOPMENT OF THE FREDHOLM EQUATION \\ CONTAINING THE SIMPLE TEMPERATURE DISTRIBUTION FUNCTION FOR SYSTEMS WITH TEMPERATURE EXTREMA}

A plug-flow system consisting of two consecutive sections is postulated. In the first portion, Section A, the temperature rises monotonically. In the second portion, Section B, the temperature decreases monotonically.

$$
\text { To prove: } \int_{0}^{1} e^{-E / R T} d \xi=\int_{0}^{1} e^{-E / R T} \eta(T) d T \text {. }
$$

If this can be proven, substitution into Eq. (III-14) yields Eq. (V-3). The solution to the general situation, in which the temperature can rise or fall any number of times within the system, will be obvious after treatment of this special case.

To prove the special case, one form of Eq. (V-2) is first inverted, giving

$$
\left|\frac{d T}{d V}\right|=\frac{1}{V_{s} \eta(T)}
$$

The sign attached to the temperature slope $\mathrm{dT} / \mathrm{dV}$ depends on whether the temperature is rising or falling within the system. In Section A of our postulated system, it is rising, so

$$
\left.\frac{\mathrm{dT}}{\mathrm{dV}}\right|_{\mathrm{A}}=\frac{1}{\mathrm{~V}_{\mathrm{s}} \eta_{\mathrm{A}}(\mathrm{T})}
$$

In this equation, $\eta_{A}(T) d T$ is the fraction of the entire system with temperatures between $T$ and $T+d T$ that also lies within Section A. Similarly, the temperature is falling in Section B, so the slope $\mathrm{dT} / \mathrm{dV}$ is negative, and

$$
\left.\frac{d T}{d V}\right|_{B}=-\frac{1}{V_{s} \eta_{B}(T)} .
$$

Here $\eta_{B}(T) d T$ is the fraction of the entire system with temperatures between $T$ and $T+d T$ that also lies within Section B.

Let the volume of Section $A$ be $V_{A}$, and the volume of Section $B$ is then $V_{S}-V_{A}$. Multiplying both sides of Eqs. (B-3) and (B-4) by the quantity $\mathrm{e}^{-\mathrm{E} / R T}$ yields

$$
\left(e^{-E_{i} / R T}\right)\left(\frac{d T}{d V}\right)=\frac{e^{-E / R T}}{V_{s} \eta_{A}(T)}
$$

and 


$$
\left(e^{-E_{i} / R T}\right)\left(\frac{d T}{d V}\right)=\frac{e^{-E / R T}}{V_{s} \eta_{B}(T)}
$$

Multiplying through by the distribution functions, then integrating these equations between the appropriate limits, results in

$$
\begin{aligned}
& \left(\frac{1}{V_{s}}\right) \int_{0}^{V_{A}} e^{-E / R T} d V=\int_{T_{0}}^{T_{A}} e^{-E / R T} \eta_{A}(T) d T \text { for Section } A \text {, and } \\
& \left(\frac{1}{V_{s}}\right) \int_{V_{A}}^{V_{s}} e^{-E / R T} d V=-\int_{T_{A}}^{T_{s}} e^{-E / R T} \eta_{B}(T) d T \text { for Section } B .
\end{aligned}
$$

In these equations, $T_{A}$ is the temperature at the end of Section $A$ (and the beginning of Section $B$ ), and $T_{S}$ is the temperature at the end of Section $B$ (and therefore the end of the system). Letting the sum of the LHSs of Eqs. (B-7) and (B-8) equal the sum of the RHSs of these equations gives

$$
\left(\frac{1}{V_{s}}\right) \int_{0}^{V_{s}} e^{-E / R T} d V=\int_{T_{0}}^{T_{A}} e^{-E / R T} \eta_{A}(T) d T-\int_{T_{A}}^{T_{s}} e^{-E / R T} \eta_{B}(T) d T .
$$

In the integrals on the RHS of Eq. (B-9), the limits can be extended to any points below $T_{0}$ and $T_{s}$ without changing the value of the integrals. The function $\eta_{A}(T)$ is zero at any point below $T_{0}$ and $\eta_{B}(T)$ is zero at any point below $T_{s}$. Similarly, the $T_{A}$ limit can be set to any point above $T_{A}$ without changing the values of the integrals, since both $\eta_{A}(T)$ and $\eta_{B}(T)$ are zero at all temperatures above $T_{A}$. Using these properties, the low temperature limits are set at 0 , and the upper temperature limits at infinity. Doing this changes Eq. (B-9) to

$$
\left(\frac{1}{V_{s}}\right) \int_{0}^{V_{s}} e^{-E / R T} d V=\int_{0}^{\infty} e^{-E / R T} \eta_{A}(T) d T-\int_{\infty}^{0} e^{-E / R T} \eta_{B}(T) d T .
$$

Reversing the limits in the second integral on the RHS of Eq. (B-10), then adding the two integrals on this side, simplifies the equation to

$$
\left(\frac{1}{V_{S}}\right) \int_{0}^{V_{s}} e^{-E / R T} d V=\int_{T_{0}}^{T_{A}} e^{-E / R T}\left[\eta_{A}(T)+\eta_{B}(T)\right] d T .
$$

Because $\eta_{A}(T)+\eta_{B}(T)=\eta(T)$,

$$
\left(\frac{1}{V_{s}}\right) \int_{0}^{V_{s}} e^{-E / R T} d V=\int_{T_{0}}^{T_{A}} e^{-E / R T} \eta(T) d T .
$$

Because $\xi=V / V_{s}$,

$$
\left(\frac{1}{V_{s}}\right) \int_{0}^{V_{s}} e^{-E / R T} d V=\int_{0}^{V_{s}} e^{-E / R T} \frac{d V}{V_{s}}=\int_{0}^{1} e^{-E / R T} d \xi .
$$

The integral limits on the RHS of Eq. (B-13) can be changed from 0 to $T_{\min }$ and from $\infty T_{\max }$ because $\eta(T)$ is zero in these intervals and the value of the integral remains unaltered by these changes in 
limits. Changing the limits on the RHS of Eq. (B-13) and substituting the RHS of Eq. (B-14) for the LHS of Eq. (B-13) gives

$$
\int_{0}^{1} e^{-E R T} d \xi=\int_{0}^{1} e^{-E / R T} \eta(T) d T
$$

This is the desired proof for a two-section plug-flow system. The same approach can be used for a system of any number of sections. Thus, if there were twenty changes of temperature-volume slope within a system, then twenty sections would be considered in the proof, and the result would be identical with Eq. (B-1). The same approach also can be used for the temperature histories of batch systems, with the temperature rising and falling throughout the history of the system. 


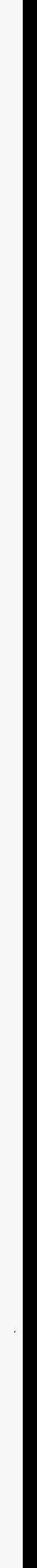




\section{APPENDIX C \\ EXPRESSIONS FOR MATRICES AND VECTOR IN EQ. (VII-5)}

Here, two different stabilizers are discussed. For both of them, the matrix $\mathbf{M}$ and the vector $\underline{\mathbf{h}}$ are defined as follows:

$$
\begin{aligned}
\underline{M}(j, k) & =\Delta y_{k} \int_{E_{\min }}^{E_{\max }} K\left(E, y_{j}\right) K\left(E, y_{k}\right) d E, \text { or } \\
& =\Delta y_{k} \sum_{i=1}^{n} K\left(E_{i}, y_{i}\right) K\left(E_{i}, y_{k}\right) \Delta E_{i} ; \\
h(j) & =\sum_{i=1}^{n} K\left(E_{i}, y_{j}\right) g\left(E_{i}\right) \Delta E_{i} .
\end{aligned}
$$

The definition of matrix $\mathbf{H}$ depends on the type of stabilizer used.

Case I : $G(f)=[f(y)]^{2}$

$\mathbf{H}$ is defined as the identity matrix.

Case II : $G(f)=q_{0}[f(y)]^{2}+q_{1}[d f(y) / d y]^{2}$

$\mathbf{H}$ is uniformly zero, except for the following elements:

$$
H(i, j)=\begin{array}{ll}
q_{0}+q_{1} /\left(\Delta y_{i}\right)^{2} & \text { if } i=j=1, \text { or } i=j=m ; \\
q_{0}+2 q_{1} /\left(\Delta y_{i}\right)^{2} & \text { if } i=j \text {, and } i \neq 1 \text { or } m ; \\
-q_{1} /\left(\Delta y_{i}\right)^{2} & \text { if }|i-j|=1 .
\end{array}
$$

When the boundary conditions [i.e., $f\left(y_{1}\right)$ and $f\left(y_{m}\right)$ ] are specified, we need to solve only $(m-2)$ equations, instead of $\mathrm{m}$ equations. In that case, the vector $\underline{h}$ is modified using the first and the last columns of $\mathbf{M}$ and $\mathbf{H}$. Then, by ignoring the first and the last columns and rows of $\mathbf{M}$ and $\mathbf{H},(\mathrm{m}-2)$ equations can be solved to obtain $f\left(y_{j}\right)$ for $j=2$ to $m-1$. 




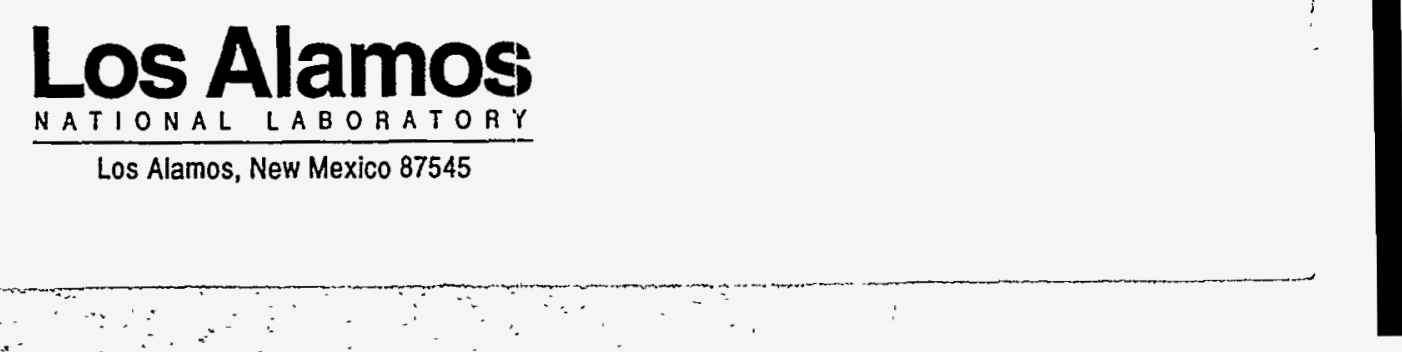

